\title{
Perception affects the brain's metabolic response to sensory stimulation
}

\section{Short title: Cortical metabolic response to visual perception}

Mauro DiNuzzo ${ }^{1, \S}$, Silvia Mangia ${ }^{2,}$, Marta Moraschi $^{3}$, Daniele Mascali ${ }^{1,4}$, Gisela E. Hagberg ${ }^{5}$, Federico Giove ${ }^{1,6, *}$

${ }^{1}$ Museo Storico della Fisica e Centro Studi e Ricerche Enrico Fermi, 00184 Rome, Italy

${ }^{2}$ Center for Magnetic Resonance Research, Department of Radiology, University of Minnesota, Minneapolis, MN 55455, USA

${ }^{3}$ Unità Operativa di Radioterapia Oncologica, Università Campus Bio-Medico, 00128 Rome, Italy

${ }^{4}$ Dipartimento di Neuroscienze, Imaging e Scienze Cliniche, Università Gabriele D’Annunzio, 66100 Chieti, Italy

${ }^{5}$ High-Field Magnetic Resonance, Max Planck Institute for Biological Cybernetics and Biomedical Magnetic Resonance, University Hospital Tübingen, 72076 Tübingen, Germany

${ }^{6}$ Fondazione Santa Lucia IRCCS, 00179 Rome, Italy

$\S$ These authors contributed equally to this work.

\author{
* correspondence to: \\ Federico Giove, Ph.D. \\ Magnetic Resonance for Brain Investigation Laboratory \\ c/o Fondazione Santa Lucia \\ Via Ardeatina 354, 00179 Rome, Italy \\ Phone: +3906 51501157 \\ mail: federico.giove@cref.it
}




\section{Author contributions}

2 Conceptualization and Methodology: MDN, SM, GEH and FG. Investigation: MDN, DM, MM

3 and FG. Data curation and Formal analysis: MDN, DM, MM and FG. Writing - original draft:

4 MDN, SM and FG. Writing - review and editing: All authors. Supervision: FG.

\section{Competing interests}

6 The authors declare no competing financial interests.

8 Keywords: central visual system $\bullet$ visual perception $\bullet$ isoluminant chromatic flickering $\bullet$ human

9 brain metabolism $\bullet$ lactate $\cdot$ BOLD fMRI $\bullet$ single voxel ${ }^{1} \mathrm{H}$-fMRS

10 Abstract word count: 190

11 Word count: about 5400 (excluding abstract, references and figure legends).

12 Number of Tables: 3

13 Number of figures: 4

14 References count: 95

15 Supplementary Information: 5 Figures (as supplements to main text figures), 1 Movie.

16

17 Article type: Research Article 


\title{
18 Authors Contact Information
}

\author{
Mauro DiNuzzo \\ ORCiD: https://orcid.org/0000-0003-0181-5597 \\ email: mauro.dinuzzo@neuroenergetics.org \\ Silvia Mangia \\ ORCiD: https://orcid.org/0000-0001-6341-4516 \\ email: mangia@umn.edu \\ Marta Moraschi \\ ORCiD: https://orcid.org/0000-0003-4789-6636 \\ email:martamoras@gmail.com \\ Daniele Mascali \\ ORCiD: https://orcid.org/0000-0003-1269-6060 \\ email: daniele.mascali@unich.it \\ Gisela E. Hagberg \\ ORCiD: https://orcid.org/0000-0003-2176-7086 \\ email: gisela.hagberg@tuebingen.mpg.de \\ Federico Giove \\ ORCiD: https://orcid.org/0000-0002-6934-3146 \\ email: federico.giove@,cref.it
}


Processing of incoming sensory stimulation triggers an increase of cerebral perfusion and blood oxygenation (neurovascular response) as well as an alteration of the metabolic neurochemical

21 profile (neurometabolic response). Here we show that perceived and unperceived isoluminant

22 chromatic flickering stimuli designed to have similar neurovascular responses as measured by

23 blood oxygenation level dependent functional MRI (BOLD-fMRI) in primary visual cortex (V1)

24 have markedly different neurometabolic responses as measured by functional MRS. In particular,

25 a significant regional buildup of lactate, an index of aerobic glycolysis, and glutamate, an index of

26 malate-aspartate shuttle, occurred in V1 only when the flickering is perceived, without any relation

27 with behavioral or physiological variables. Wheras the BOLD-fMRI signal in V1, a proxy for input

28 to V1, was insensitive to flickering perception by design, the BOLD-fMRI signal in secondary

29 visual areas was larger during perceived than unperceived flickering indicating increased output

30 from V1. These results indicate that the upregulation of energy metabolism induced by visual

31 stimulation depends on the type of information processing taking place in $\mathrm{V} 1$, and that $1 \mathrm{H}-\mathrm{fMRS}$

32 provides unique information about local input/output balance that is not measured by BOLD-

33 fMRI.

\section{Significance statement:}

35 Visual perception has a measurable metabolic effect in the primary visual cortex (V1). Here we

36 show that the upregulation of energy metabolism induced by isoluminant chromatic flickering

37 depends on subjective visual perception. Within V1, perceived and unperceived stimuli that are

38 contrast-matched to elicit similar blood-oxygenation level-dependent fMRI response are

39 associated with clearly distinct neurochemical profiles. Specifically, regional accumulations of

40 lactate (an index of aerobic glycolysis) and glutamate (an index of malate-aspartate shuttle) only 
41 develop during perceived stimuli, together with a larger activation of secondary visual areas. Our

42 results imply a dissociation between metabolic and functional response, and indicate that that the

43 upregulation of energy metabolism induced by visual stimulation depends on the type of

44 information processing taking place in V1. 


\section{Introduction}

46 In the brain, sensory stimulation is associated with a substantial increase of regional functional

47 hyperaemia (i.e. cerebral blood flow, CBF) as well as energy metabolism of glucose, the main

48 cerebral energy substrate (1). The metabolic response to stimulation includes an oxidative

49 component, as measured by the cerebral metabolic rate of oxygen $\left(\mathrm{CMRO}_{2}\right)$, and a non-oxidative

50 component, as reflected by lactate accumulation (2). Cortical lactate levels have been shown to

51 increase during visual stimulation, simultaneously to the acceleration of the malate-aspartate

52 shuttle, a process termed aerobic glycolysis (i.e. lactate production independent of oxygen

53 availability) (3-11). Despite intense research, the neurophysiological mechanisms underlying the

54 up-regulation of glycolytic metabolism of glucose are still largely unknown (12). Most

55 importantly, the impact of information processing to the metabolic response of the cerebral cortex

56 to sensory stimulation has not been fully investigated thus far. In particular, nothing is known about

57 the modulatory effect exerted by the perception of different stimuli on regional brain energy

58 metabolism.

59 Sensory perception is thought to rely on the complex interplay of neural circuits that process

60 information in a cortical layer- and area-mediated manner involving thalamo-cortical, intracortical,

61 cortico-cortical and cortico-thalamic feedforward/feedback loops (13). Sensory stimuli transduced

62 by sensory organs reach specific thalamic nuclei that relay information to primary sensory cortices,

63 which in turn filter and eventually transmit information to secondary sensory areas (14). These

64 transactions are dependent on the particular features of different incoming stimuli, thus it is

65 possible that the relevant neurovascular and neurometabolic counterparts are correspondingly

66 distinct (15). 
67 The thalamic lateral geniculate nucleus (LGN) mediates visual stimuli with temporal frequencies

68 at least up to $90 \mathrm{~Hz}$ to the layer IV of V1 (16-21), which in turn relays to output layers II/III and

$69 \mathrm{~V}$ where temporal filtering occurs (22), consistent with the notion that visual perception requires

70 the activation of visual areas downstream V1 (i.e. secondary visual cortices). In agreement with

71 these arguments, it has been repeatedly reported that invisible visual flickering is still able to

72 activate V1 even without any perceptual effects (23), as revealed by in vivo electrophysiology in

73 non-human primates (16) as well as behavioral evidence (24) and BOLD fMRI (25) in humans.

74 High $(30 \mathrm{~Hz})$ frequency visual stimulation has been found to selectively suppress multi-unit

75 activity (MUA) in cat V1 as compared to low frequency (4 Hz) visual stimulation (26).

76 Importantly, local field potentials (LFPs) and tissue oxygen response, which directly contribute to

77 the generation of the BOLD signals (27), were preserved at both frequencies.

78 In the present study, we combined blood-oxygenation level dependent (BOLD) functional

79 magnetic resonance imaging (fMRI) and proton functional magnetic resonance spectroscopy (1H-

80 fMRS) in humans and exploited the well known effect of temporal frequency on visual perception.

81 Specifically, we examined the functional and metabolic responses of the primary visual cortex

82 (V1) to perceived or unperceived isoluminant chromatic flickering stimulations obtained by using

83 temporal frequency either below (7.5 Hz; PF, perceived flickering) or above (30 Hz; UF,

84 unperceived flickering) the critical flicker fusion (CFF) threshold of $\sim 15 \mathrm{~Hz}$ for rod-mediated

85 vision (28). Based on experimental evidence and metabolic modeling, we have previously

86 proposed that the local input-output balance between neuronal synaptic/spiking (or

87 subthreshold/suprathreshold) activity is a primary determinant in the up-regulation of aerobic

88 glycolysis (29-31). We thus hypothesized that the loss of visual perception is accompanied by

89 fundamental changes in the metabolic responses of human V1. 


\section{$91 \quad$ Results}

\section{Subjects perception of the visual stimuli}

93 To achieve perceptual isoluminance between green and red color (necessary for loss of perception

94 at $30 \mathrm{~Hz}$ ), we adjusted the brightness of the green color for each individual subject, which was

95 remarkably similar across subjects (green/red brightness ratio $71.9 \pm 1.2 \%$, range $70.1 \%$ to $73.5 \%$;

96 see Table 1). After this procedure, $100 \%$ of the subjects confirmed that their perception of the 30

$97 \mathrm{~Hz}$ frequency stimulus steadiness was equivalent to the resting condition. Overall, the subject's

98 perception was a gray/colored checkerboard that in the colored squares showed either a fast green

99 and red alternation during PF epochs, or a static yellow during UF epochs (Movie S1). As a further

100 confirmation, while in the scanner the subjects were unable to distinguish the $30 \mathrm{~Hz}$ red-green

101 flickering checkerboard (used in the actual experiments) from a color-matched static yellow

102 checkerboard (used for testing only). Specifically, the perception of the steady yellow color versus

103 the $30 \mathrm{~Hz}$ red-green flickering was indistinguishable, as assessed by asking the subjects to guess

104 the origin of the stimulus for 10 consecutive trials (average of correct responses $52 \pm 16 \%$, not

105 different from chance level, $\mathrm{p}=0.62$ ). All subjects reported to distinctly perceive the green and red

106 color when the checkerboard was flickering at $7.5 \mathrm{~Hz}$. None of the subject perceived the intrinsic

107 flickering of the screen due to the refresh rate $(60 \mathrm{~Hz})$. 
Table 1. Demographics and study parameters.

\begin{tabular}{|c|c|c|c|c|c|c|c|}
\hline \multirow[b]{2}{*}{ Count } & \multirow[b]{2}{*}{ Gender } & \multirow[b]{2}{*}{ Age } & \multicolumn{2}{|c|}{ fMRI } & \multicolumn{3}{|c|}{ 1H-fMRS } \\
\hline & & & Calibration & Run & Voxel & Runs & $\begin{array}{c}\text { Water } \\
\text { Linewidth }(\mathrm{Hz})\end{array}$ \\
\hline 1 & M & 35 & 72.5 & 1 & $\mathrm{~L}$ & $1 / 2$ & 6.8 \\
\hline 2 & $\mathrm{~F}$ & 32 & 72.5 & 2 & $\mathrm{~L}$ & $2 / 1$ & 8.3 \\
\hline 3 & M & 29 & 73.5 & 1 & $\mathrm{R}$ & $1 /-$ & 7.5 \\
\hline 4 & F & 24 & 71.8 & 2 & $\mathrm{R}$ & $2 / 1$ & 7.7 \\
\hline 5 & M & 26 & 72.1 & 1 & $\mathrm{~L}$ & $1 / 2$ & 6.7 \\
\hline 6 & $\mathrm{~F}$ & 27 & 73.5 & 2 & $\mathrm{~L}$ & $2 / 1$ & 6.7 \\
\hline 7 & M & 28 & 70.1 & 1 & $\mathrm{~L}$ & $1 / 2$ & 5.7 \\
\hline 8 & M & 20 & 72.1 & 1 & $\mathrm{R}$ & $2 / 1$ & 7.5 \\
\hline 9 & $\mathrm{~F}$ & 26 & 70.1 & 2 & $\mathrm{~L}$ & $1 / 2$ & 6.6 \\
\hline 10 & $\mathrm{~F}$ & 28 & 72.1 & 1 & $\mathrm{R}$ & $2 / 1$ & 6.8 \\
\hline 11 & $\mathrm{~F}$ & 20 & 70.1 & 2 & $\mathrm{R}$ & $1 / 2$ & 7.5 \\
\hline 12 & $\mathrm{~F}$ & 20 & 73.5 & 1 & $\mathrm{~L}$ & $1 / 2$ & 7.9 \\
\hline 13 & $\mathrm{~F}$ & 20 & 71.8 & 1 & $\mathrm{R}$ & $2 / 1$ & 7.8 \\
\hline 14 & M & 21 & 73.5 & 2 & $\mathrm{~L}$ & $2 / 1$ & 7.4 \\
\hline 15 & M & 21 & 71.8 & 1 & $\mathrm{~L}$ & $2 / 1$ & 7.6 \\
\hline 16 & F & 26 & 70.1 & 2 & $\mathrm{R}$ & $2 / 1$ & 7.5 \\
\hline 17 & M & 20 & 70.1 & 1 & $\mathrm{R}$ & $2 / 1$ & 7.0 \\
\hline 18 & $\mathrm{~F}$ & 26 & 72.8 & 2 & $\mathrm{R}$ & $1 / 2$ & 6.3 \\
\hline 19 & M & 25 & 71.8 & 1 & $\mathrm{~L}$ & $2 / 1$ & 7.1 \\
\hline
\end{tabular}

109

110 Calibration refers to the \% brightness adjustment for the green color relative to the brightness of the red color. A dash

111 indicates that the relevant measurement has not been performed due to technical reasons.

\section{Subjects attention}

113 To examine the possible modulation of the BOLD signal by attention (32), we measured

114 pupillometry and task-performance data during all experiments. All subjects maintained gaze on

115 the fixation cross during the whole epochs (Figure 1A), with no difference in average gaze location 
116 between the different stimulations (Figure 1B,C and Figure 1 - figure supplement $1^{1}$ ). Pupil

117 diameter, an index of the noradrenergic tone (33), was fairly stable across conditions (Figure 1D,E;

118 Figure 1 - figure supplement 2 and Movie S1), indicating that the modulation of perception by

119 noradrenaline (34) was minimal in our experimental conditions.

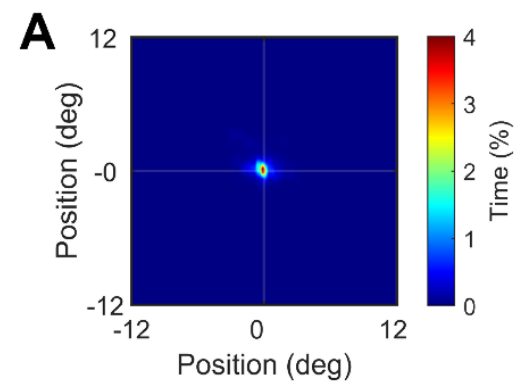

D

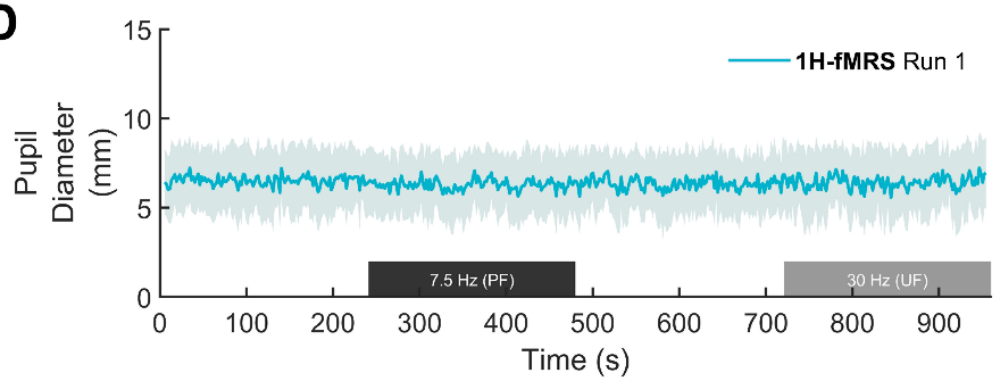

B

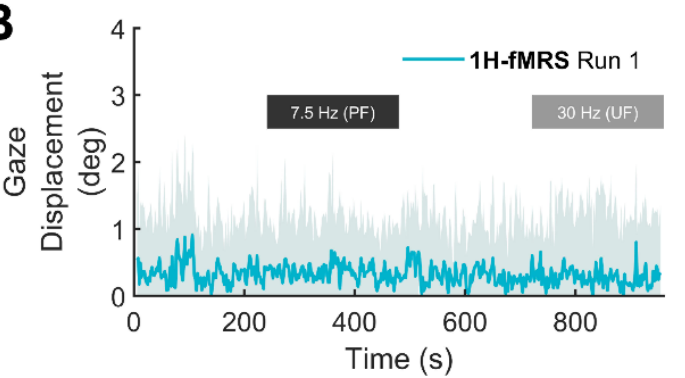

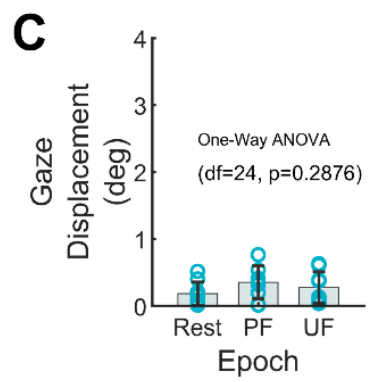

$\mathbf{E}$

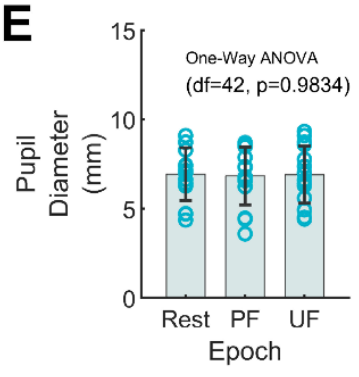

$\mathbf{F}$

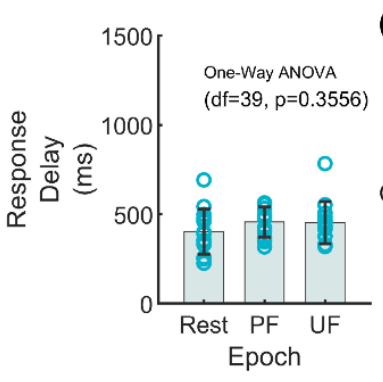

G $\quad 1.5$

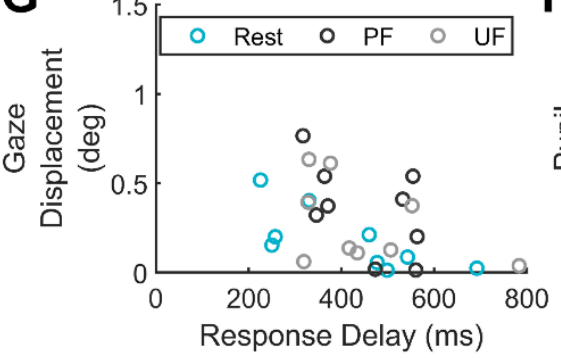

H

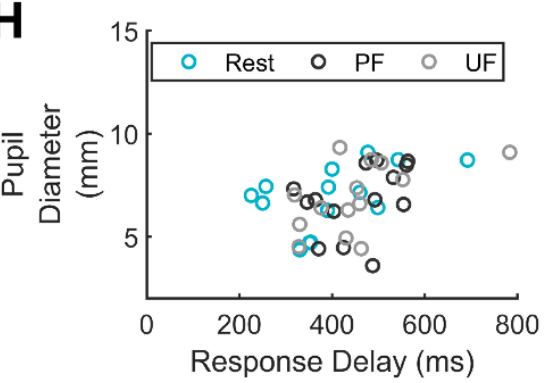

121 Figure 1. Subjects perception and attention during visual stimulation. (A) Average heatmap of eyes position

122 (across subjects) during a representative session (1H-fMRS Run 1). (B) Stability of mean gaze displacement from the

123 fixation point. (C) Gaze displacement was not different across conditions (One-Way ANOVA, p=0.29). Error bars

124 correspond to SD. (D) Average pupil diameter (across-subjects) during a representative session (1H-fMRS Run 1). (E)

125 Mean pupil diameter was not statistically different across conditions (One-Way ANOVA, p=0.98). Error bars

126 correspond to SD. (F) Task performance in terms of response delay was not statistically different across conditions

127 (One-Way ANOVA, $\mathrm{p}=0.36$ ). Error bars correspond to SD. (G) There was no correlation between task performance

\footnotetext{
${ }^{1}$ [Note for reviewing only] Following stile suggesiton of eLife, supplementary figures are linked as children to a main figure. Figure supplements and the relevant captions are at the end of this file.
} 
and mean gaze displacement $\left(\mathrm{r}^{2}<0.06, \mathrm{p}>0.47\right)$. (H) There was no correlation between task performance and mean pupil diameter $\left(\mathrm{r}^{2}<0.08, \mathrm{p}>0.49\right)$.

131 The hit/miss ratio for the cross rotation task during the stimulation protocol was essentially 1.0 , as

132 expected due to the simplicity of the task, for the entire duration of the experiment. In particular, 133 the delay of the response was not statistically different for rest, PF and UF epochs (ranging, on 134 average, between $360 \mathrm{~ms}$ and $460 \mathrm{~ms}$ ), both during fMRI (One-Way ANOVA, $\mathrm{p}=0.09$ and $\mathrm{p}=0.77$ 135 for run 1 and 2, respectively) and 1H-fMRS (One-Way ANOVA, $p=0.39$ and $p=0.51$ for run 1 and 1362 , respectively), confirming high and similar levels of subject's attention across conditions (Figure

$137 \quad 1 \mathrm{~F}$ and Figure 1 - figure supplement 3 ). There was no significant correlation between task 138 performance and mean pupil diameter or gaze displacement $(\mathrm{p}>0.21$ and $\mathrm{p}>0.34$, respectively;

139 Figure 1G,H and Figure 1 - figure supplement 3). Finally, in-scanner head motion during fMRI 140 scans was minimal and no significantly different for all subjects across epochs (mean framewise 141 displacement $0.25 \pm 0.12 \mathrm{~mm}$ for rest, $0.24 \pm 0.14 \mathrm{~mm}$ for PF, $0.23 \pm 0.08 \mathrm{~mm}$ for UF; One-Way 142 ANOVA, $\mathrm{p}=0.77)$. Overall, behavioral and physiological variables associated with attentional load 143 were maintained at considerably constant levels in all subjects.

\section{Similar BOLD responses in V1 to perceived and unperceived flickering}

145 To achieve the same BOLD response in V1 during PF and UF, we reduced the stimulation contrast 146 for the $7.5 \mathrm{~Hz}$ condition to $75 \%$ relative to the $30 \mathrm{~Hz}$ condition (Figure $2 \mathrm{~A}$ ). As expected, we found 147 that the average BOLD timecourse (Figure 2B) as well as the change in the subject-matched 148 spectroscopic VOI (on average consisting of $47 \pm 9 \%$ of BA17, $21 \pm 12 \%$ of BA18, and $16 \pm 9 \%$ of 149 BA19; see Figure 2 - figure supplement 1), was similar between the two conditions $(0.44 \pm 0.30 \%$ 150 for PF versus $0.41 \pm 0.25 \%$ for $\mathrm{UF}$, paired two-sample t-test, $\mathrm{p}=0.71$ ) (Figure $2 \mathrm{C}$ ). The fMRI 151 activations to PF and UF both peaked in V1 and distinctly spanned bilaterally in secondary visual 
bioRxiv preprint doi: https://doi.org/10.1101/2021.09.17.460759; this version posted September 20, 2021. The copyright holder for this preprint (which was not certified by peer review) is the author/funder, who has granted bioRxiv a license to display the preprint in perpetuity. It is made available under aCC-BY 4.0 International license.

152 areas (Figure 2D,E, one-sample t-test, FDR-corrected at cluster level, $\mathrm{q}<0.05$, voxel level $153 \mathrm{p}<0.001)$.

154 

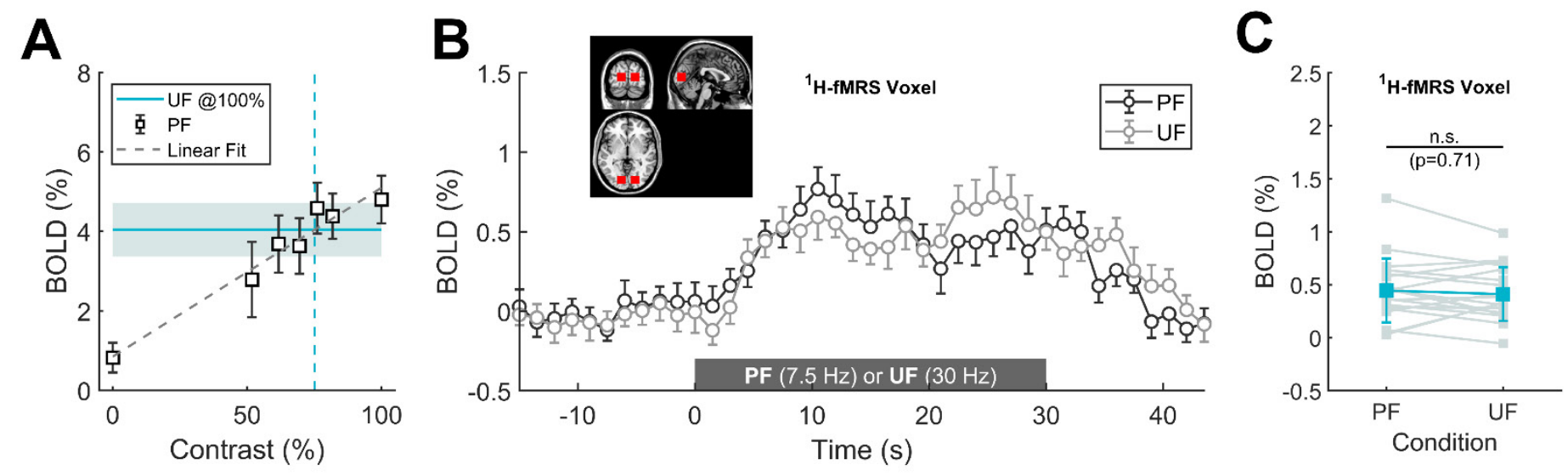

\section{D}
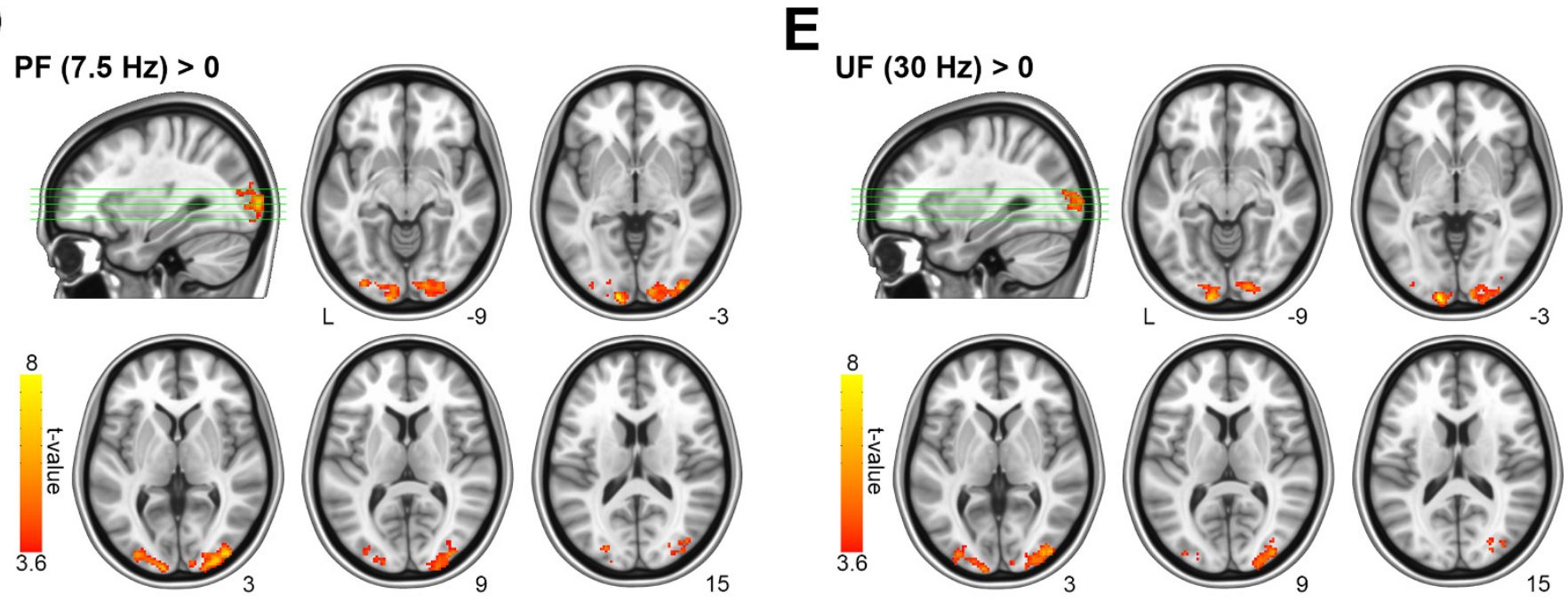

Figure 2. Main effect of stimulation assessed by fMRI analysis. (A) Calibration of image contrast to match BOLD

157 response in V1 to PF and UF obtained in a preliminary session. The contrast of the PF image was reduced to $75 \%$ for

158 subsequent stimulations (i.e. common to all subjects). (B) Mean time-course of BOLD signals in the transition between

159 rest and PF or rest and UF, averaged over the fMRI voxels corresponding to the subject-specific spectroscopic VOI.

160 (C) BOLD percent change during the experimental conditions, averaged over the fMRI voxels corresponding to the

161 subject-specific spectroscopic VOI. No statistically significant difference in BOLD response was found between the

162 two conditions (unpaired two-sample t-test, $p>0.71$ ). (D,E) Statistical maps for group-averaged positive effect of the

163 visual stimulation (PF and UF) versus rest. Normalized maps are thresholded at $\mathrm{p}<0.001$, with a FDR correction at the

164 cluster level (corresponding to $\mathrm{q}<0.05$ ), and overlaid on MNI template. 


\section{Different BOLD responses in secondary visual areas to perceived and unperceived}

\section{6 flickering}

167 To better characterize the effect of the two different stimulations, we estimated the main effect of

168 the flickering frequency. The main effect of PF appeared in the lateral occipital cortices, but not in

169 V1 (Figure 3B and Table 2). Although the electrophysiological activity in V1 could not be directly

170 assessed in our experiment, based on the literature (27) we can obtain a rough surrogate of V1

171 output by evaluating fMRI signals in the secondary visual areas, which receive input directly from

172 V1. The average BOLD change in these areas (Brodmann Areas 18 and 19) was significantly

173 higher during PF than UF $(0.61 \pm 0.29 \%$ versus $0.39 \pm 0.18 \%$, paired two-sample t-test, $\mathrm{p}=0.008)$,

174 while the response in V1 (Brodmann Area 17) was similar for the two stimulations $(0.85 \pm 0.45 \%$

175 versus $0.80 \pm 0.42 \%$, paired two-sample t-test, $\mathrm{p}=0.72$ ) (Figure $2 \mathrm{C}, \mathrm{D}$ ), indicating a larger output

176 from V1 during PF compared with UF. Thus, V1 exhibited the same BOLD signal despite known

177 differences in visual processing for PF and UF (26). 
A
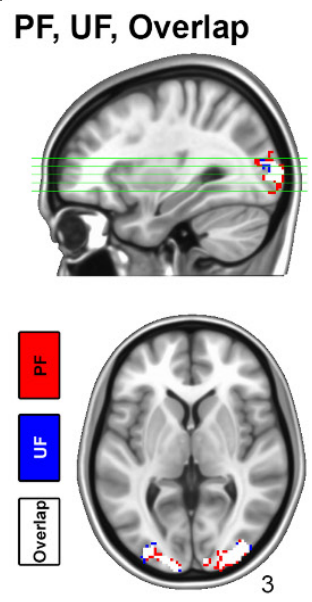
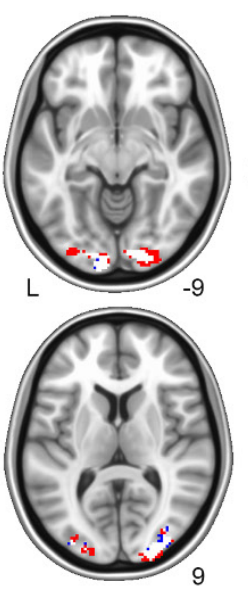
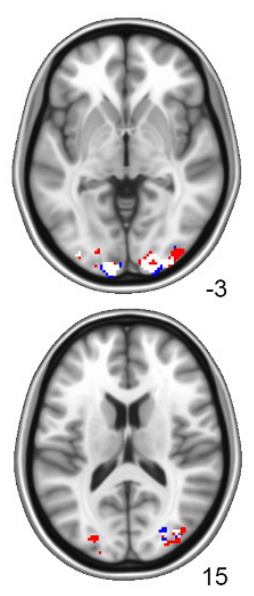

B

PF $(7.5 \mathrm{~Hz})>$ UF $(30 \mathrm{~Hz})$

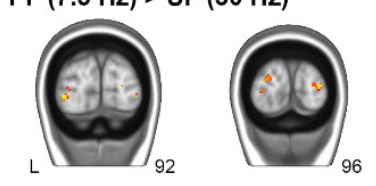

C

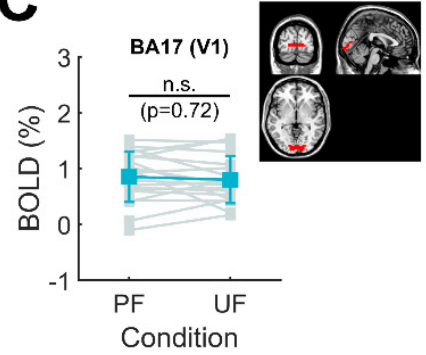

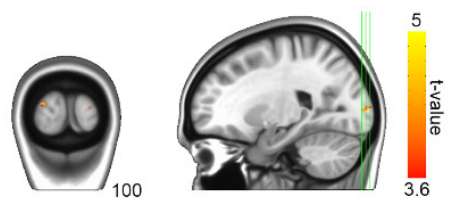

D

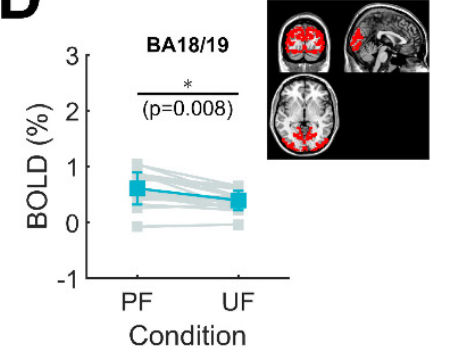

180 Figure 3. Differential effect of stimulation assessed by fMRI analysis. (A) Overlap between activation maps

181 relative to PF and UF. (B) Differential effect of stimulation ( $P F>U F)$. Statistics are thresholded as in Figure $2(\mathrm{p}<0.001$,

$182 \mathrm{q}_{\mathrm{FDR}}<0.05$ ). The differential response related to perception is localized in lateral occipital cortex (secondary visual

183 areas), with no responding voxels inside V1. For comparison, the inverse differential effect of stimulation (UF>PF)

184 has no significant responding voxels (not shown). (D) BOLD percent change averaged over the fMRI voxels

185 corresponding to the Brodman Area 17 (i.e., V1). Within V1, there is no difference between PF and UF condition

186 (unpaired two-sample t-test, $\mathrm{p}=0.72$ ). (E) BOLD percent change averaged over the fMRI voxels corresponding to the

187 Brodman Areas 18 and 19 (e.g., including V2, V3a, V4v, V5/MT). Within these areas, the response to PF is

188 significantly larger than the corresponding response to UF (unpaired two-sample t-test, $\mathrm{p}=0.008$ ).

190 Table 2. Cortical regions preferentially activated by PF compared to UF.

\begin{tabular}{|c|c|c|c|c|c|c|c|}
\hline \multirow[t]{2}{*}{ Cluster } & \multirow{2}{*}{\multicolumn{2}{|c|}{ Anatomical Label }} & \multirow{2}{*}{$\begin{array}{c}\text { Size } \\
\text { (Voxels) }\end{array}$} & \multirow[t]{2}{*}{ qFDR } & \multicolumn{3}{|c|}{$\begin{array}{l}\text { Coordinates } \\
(\mathrm{mm})\end{array}$} \\
\hline & & & & & $X$ & $\mathrm{Y}$ & $\mathrm{Z}$ \\
\hline \multirow[t]{2}{*}{1} & Peak & $\begin{array}{l}\text { 45.0\% GM Visual cortex V3V L } \\
\text { 40.0\% GM Visual cortex V4 L } \\
\text { 10.0\% GM Visual cortex V2 BA18 L }\end{array}$ & 28 & 0.003 & -32 & -92 & -6 \\
\hline & ROI & $\begin{array}{l}\text { 50\% GM Visual cortex V3V L } \\
\text { 39\% GM Visual cortex V4 L }\end{array}$ & & & & & \\
\hline 2 & Peak & $\begin{array}{l}\text { 53.0\% GM Visual cortex V3V R } \\
\text { 19.0\% GM Visual cortex V2 BA18 } \\
\text { R } \\
9.0 \% \text { GM Visual cortex V1 BA17 R }\end{array}$ & 25 & 0.003 & 30 & -96 & 6 \\
\hline
\end{tabular}




\begin{tabular}{|c|c|c|c|c|c|c|c|}
\hline & ROI & $\begin{array}{l}48 \% \text { GM Visual cortex V3V R } \\
24 \% \text { GM Visual cortex V1 BA17 R } \\
16 \% \text { GM Visual cortex V2 BA18 R } \\
12 \% \text { WM Optic radiation R }\end{array}$ & & & & & \\
\hline \multirow[t]{2}{*}{3} & Peak & $\begin{array}{l}\text { 40.0\% GM Visual cortex V2 BA18 L } \\
13.0 \% \text { GM Visual cortex V3V L } \\
10.0 \% \text { GM Visual cortex V1 BA17 L }\end{array}$ & \multirow[t]{2}{*}{25} & \multirow[t]{2}{*}{0.003} & \multirow[t]{2}{*}{-22} & \multirow[t]{2}{*}{98} & \multirow[t]{2}{*}{12} \\
\hline & ROI & $84 \%$ GM Visual cortex V2 BA18 L & & & & & \\
\hline \multirow[b]{2}{*}{4} & Peak & $\begin{array}{l}30.0 \% \text { GM Visual cortex V4 R } \\
10.0 \% \text { GM Visual cortex V3V R }\end{array}$ & \multirow[b]{2}{*}{14} & \multirow[b]{2}{*}{0.021} & \multirow[b]{2}{*}{42} & \multirow[b]{2}{*}{-86} & \multirow[b]{2}{*}{-2} \\
\hline & ROI & $\begin{array}{l}\text { 64\% GM Visual cortex V3V R } \\
29 \% \text { GM Visual cortex V4 R }\end{array}$ & & & & & \\
\hline \multirow[t]{2}{*}{5} & Peak & $\begin{array}{l}\text { 1.0\% GM Visual cortex V2 BA18 L } \\
1.0 \% \text { GM Visual cortex V3V L }\end{array}$ & \multirow[t]{2}{*}{11} & \multirow[t]{2}{*}{0.034} & \multirow[t]{2}{*}{-28} & \multirow[t]{2}{*}{-90} & \multirow[t]{2}{*}{4} \\
\hline & ROI & $27 \%$ WM Optic radiation L & & & & & \\
\hline
\end{tabular}

192 Cluster location was determined from either the Peak coordinate or the average ROI composition in the Juelich 193 Histological Atlas (35) using the mni2atlas tool (https://github.com/dmascali/mni2atlas).

195 Dissociation between metabolic and BOLD responses in V1 during loss of visual perception

196 To assess whether metabolism was sensitive to stimulus processing within V1 we performed 197 single-voxel 1H-fMRS. The reproducible BOLD response in the occipital lobe elicited by the PF 198 and UF stimulations allowed a very accurate VOI positioning for $1 \mathrm{H}-\mathrm{fMRS}$. High quality and 199 artifact-free spectra (water linewidth $7.2 \pm 0.6 \mathrm{~Hz}$, Figure 4A) were reliably obtained from almost 200 all subjects (Table 1 and Figure 4 - figure supplement 1). Compared with resting conditions, the 201 lactate and glutamate concentrations within V1 increased by $0.29 \pm 0.18 \mu \mathrm{mol} / \mathrm{g}$ and $0.31 \pm 0.20$ $202 \mu \mathrm{mol} / \mathrm{g}$, respectively, during the PF stimulation corresponding to an increase of about $28 \%$ and $2033 \%$, respectively, over the baseline (paired two-sample t-test, $\mathrm{q}_{\mathrm{FDR}}=0.001$ ), whereas they both 
204 remained at their basal levels $\left(-0.04 \pm 0.13 \mu \mathrm{mol} / \mathrm{g}, \mathrm{q}_{\mathrm{FDR}}=0.42\right.$ for lactate, and $0.03 \pm 0.20 \mu \mathrm{mol} / \mathrm{g}$, $205 \mathrm{q}_{\mathrm{FDR}}=0.63$ for glutamate) during the UF stimulation. The lactate and glutamate responses were

206 significantly different (paired two-sample t-test, $\mathrm{q}_{\mathrm{FDR}}=0.01$ for lactate and $\mathrm{q}_{\mathrm{FDR}}=0.003$ for 207 glutamate) among the two stimulation conditions (Figure 4B). No other metabolites among those 208 quantified showed a reliable stimulation-dependent change (Table 3). We were unable to detect a 209 reliable change for aspartate (paired two-sample t-test, $\mathrm{q}_{\mathrm{FDR}}=0.98$ ).

210 To confirm our fMRI result of similar BOLD effect during PF and UF, we examined linewidth 211 narrowing of total creatine ( $\mathrm{tCr})$ and total $\mathrm{N}$-acetylaspartate (tNAA) signals. We found a 212 stimulation-induced decrease of $\mathrm{tCr}$ and $\mathrm{tNAA}$ linewidth during both PF and UF (Figure 4C,D) 213 that was not statistically diffferent between the two conditions $(0.56 \pm 0.04 \mathrm{~Hz}$ vs $0.56 \pm 0.05 \mathrm{~Hz}$ for $214 \mathrm{tCr}$, paired-sample t-test, $\mathrm{p}=0.70 ; 0.55 \pm 0.03 \mathrm{~Hz}$ vs $0.56 \pm 0.05 \mathrm{~Hz}$ for tNAA, paired-sample t-test, $215 \mathrm{p}=0.89)$.

216 To substantiate our $1 \mathrm{H}-\mathrm{fMRS}$ result, we determined the difference spectra between conditions, 217 which mainly consisted of uncorrelated noise and only a few correlated residuals (Figure 4E).

218 Specifically, the difference between PF and rest spectra showed a signal in the region 219 corresponding to lactate, and in spectral regions corresponding to glutamate; both signals were 220 absent in the difference between UF and rest spectra. The difference spectra also featured some 221 narrow peaks corresponding to the main singlets of the spectrum, particularly $\mathrm{tCr}$ and $\mathrm{tNAA}$, as a 222 result of BOLD-induced line narrowing (36). Similar residuals on tCr and tNAA were recognizable 223 in the difference spectrum between UF and rest, but not in the difference spectrum between the 224 two active conditions, again consistent with the evidence of a similar BOLD effect on spectral 225 linewidth elicited by either of the stimulations. Overall, the only correlated signals that survived 
A

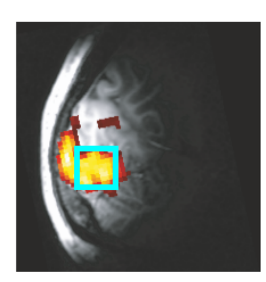

INAA

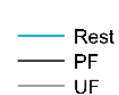

B

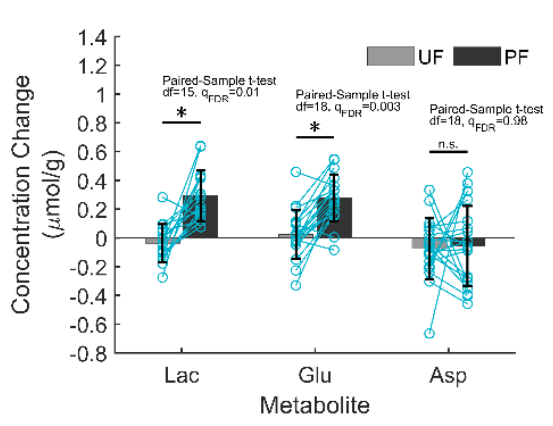

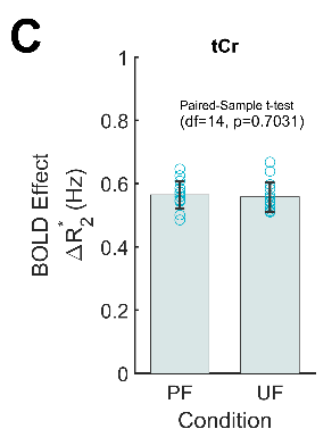

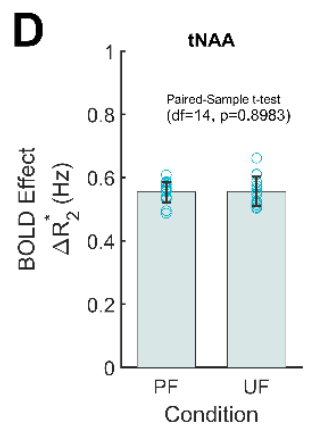

E
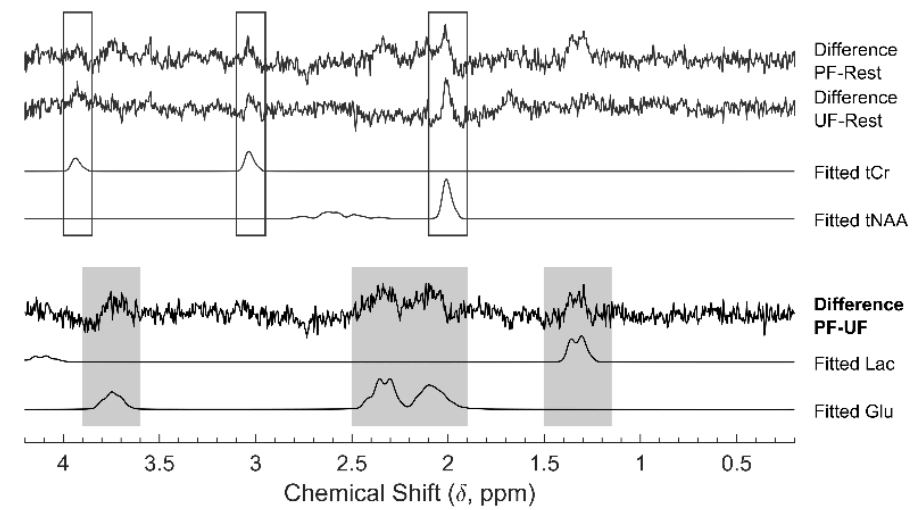

Figure 4. ${ }^{1}$ H-fMRS analysis. (A) Spectroscopic data acquired during resting condition (R, cyan) as well as PF (black) and UF (gray) averaged across subjects. A single-subject representative voxel location is reproduced on a parasagittal view of the BOLD activation and superimposed on the anatomical scan from the same subject. For visualization purposes, the processing of the spectra included frequency and phase correction of single transients, averaging, eddy currents correction, and Fourier transform. (B) Lactate, glutamate, and aspartate concentration changes during the stimulation conditions, relative to the rest conditions acquired immediately before. Data are averaged across subjects. There is significant increase in lactate $(+28 \%)$ and glutamate $(+3 \%)$ levels induced by PF stimulus, but not by UF stimulus. The concentration changes of the two metabolites were significantly different across the stimulation conditions ( $\mathrm{q}_{\mathrm{FDR}}=0.01$ for lactate and $\mathrm{q}_{\mathrm{FDR}}=0.003$ for glutamate), while there was no change for aspartate ( $\left.\mathrm{q}_{\mathrm{FDR}}=0.98\right)$. (C,D) Spectral tCr and tNAA linewidth changes induced by the PF and UF stimuli shows no statistically significant difference ( $p>0.7$ ). (E) Differences between spectra acquired in the three experimental conditions. For reference, the corresponding LCModel fits are reported on the bottom for the Lac and Glu signals. $\mathrm{tCr}$ and tNAA singlets showed the expected BOLD related features: there is a difference between stimulation and rest, but the difference spectra between the active conditions are within the noise. In the regions of lactate and glutamate the difference spectra 
244 between PF and rest and between PF and UF are similar, while they are clearly disinct from the difference spectra 245 between UF and rest.

247 Table 3. Modulations of metabolic profile of V1 during PF and UF stimulations.

\begin{tabular}{|c|c|c|c|c|}
\hline \multirow[b]{3}{*}{ Metabolite } & \multirow[b]{3}{*}{$\mathrm{N}$} & \multirow{2}{*}{$\frac{\text { CRLB (\%) }}{\text { All epochs }}$} & \multicolumn{2}{|c|}{$\Delta$ Concentration (STIM-REST) } \\
\hline & & & $\mathrm{UF}$ & $\mathrm{PF}$ \\
\hline & & CRLB (\%) & $\underset{(\mu \mathrm{mol} / \mathrm{g})}{\operatorname{mean} \pm \mathrm{SD}}$ & $\underset{(\mu \mathrm{mol} / \mathrm{g})}{\operatorname{mean} \pm \mathrm{SD}}$ \\
\hline Asp & 19 & 11 & $-0.06 \pm 0.17$ & $-0.07 \pm 0.26$ \\
\hline $\mathrm{tCr}$ & 19 & 4 & $-0.02 \pm 0.06$ & $0.00 \pm 0.08$ \\
\hline GABA & 15 & 16 & $-0.12 \pm 0.28$ & $-0.02 \pm 0.22$ \\
\hline Gln & 19 & 12 & $-0.03 \pm 0.07$ & $-0.04 \pm 0.10$ \\
\hline Glu & 19 & 5 & $0.03 \pm 0.20$ & $0.31 \pm 0.20(*)$ \\
\hline GSH & 18 & 14 & $-0.03 \pm 0.15$ & $0.06 \pm 0.12$ \\
\hline Lac & 16 & 26 & $-0.04 \pm 0.13$ & $0.29 \pm 0.18(*)$ \\
\hline Ins & 19 & 5 & $-0.02 \pm 0.09$ & $-0.01 \pm 0.11$ \\
\hline $\mathrm{NAA}+\mathrm{NAAG}$ & 19 & 3 & $-0.02 \pm 0.12$ & $0.00 \pm 0.09$ \\
\hline tCho & 19 & 7 & $0.02 \pm 0.12$ & $0.02 \pm 0.14$ \\
\hline Tau & 16 & 24 & $0.06 \pm 0.18$ & $-0.02 \pm 0.17$ \\
\hline
\end{tabular}

249 Only metabolites with CRLB $<30 \%$ in at least 15 subjects are reported ( $35 \%$ for lactate). CRLB are averaged between 250 all the epochs.

$251 \quad(*)$ : Statistically significant.

\section{Discussion}

254 The cortical gray matter of the brain features one of the highest metabolic rates of all organ tissues 255 of the human body. Although energy is recognized as a limiting factor for the human cerebral 
cortex $(37,38)$, the increase in lactate concentration occurring upon sensory stimulation isn't the

257 result of limited oxygen availability $(39,40)$, as it is for skeletal muscle. Why the cerebral cortex

258 upregulates glycolytic metabolism for sensory information processing is unknown, but it is well-

259 established that glycolysis serves specific neurophysiological and neurobiological purposes, such

260 as axonal vesicle transport, vesicle recycling, action potential waveform modulation, reuptake of

261 neuroactive compounds, and dendritic spine remodeling (reviewed in (31)). Furthermore, lactate

262 is known to be implicated in cognitive processes occurring during waking activity, like learning

263 and memory (41-44), although the exact underlying mechanisms are still debated $(45,46)$.

264 In the present study, we asked whether alterations in visual perception are also reflected in

265 metabolic changes within the primary visual cortex in humans. We report that stimulus perception

266 affects the lactate and glutamate response in V1. The PF and UF stimulations elicited, by

267 experimental design, the same average BOLD signals increase inside the spectroscopic voxel,

268 indicating an equivalent degree of neurovascular coupling and possibly of local synaptic activity

269 in the two experimental conditions (47). Yet, we observed a significant increase in the regional

270 lactate and glutamate levels only during the PF stimulus, with no appreciable change during the

271 UF stimulus compared with resting conditions. A temporal dissociation between BOLD and lactate

272 changes has been previously reported during repeated photic stimulations (48). In that case, BOLD

273 response was preserved, but not the lactate and glutamate accumulation, possibly due to

274 habituation of neuronal firing. Metabolic adaptation, in terms of glutamate levels, in the presence

275 of constant BOLD and electrophysiological activity was also reported in epilepsy (49). These

276 results suggest that the physiological mechanisms underlying BOLD signals and energy

277 metabolism do not necessarily overlap under all experimental conditions. 
278 Our results suggest that lactate and glutamate may be dissociated from BOLD changes when

279 cortical input and output are differentially modulated by e.g., intracortical inhibition (26). In fact,

280 an overall switch toward inhibition is expected to reduce the energy request of the brain, thus

281 impacting on metabolic rates independently of BOLD response (2). Accordingly, changes in

282 excitation/inhibition equilibrium have been proposed as a source of variability of the $\mathrm{CBF} / \mathrm{CMRO} 2$

283 coupling ratio (50). In more general terms, the metabolic response seems capable of differentiating

284 neural states that are intrinsically distinct, although they induce the same BOLD response. Similar

285 BOLD signal changes in correspondence to substantially different alterations in tissue lactate and

286 glutamate level during visual flickering could not be predicted a priori unless assuming

287 fundamental differences in information processing during stimulation. In particular, the firing rate

288 of layer IV neurons that receive input from LGN is higher during UF than PF, as evidenced by the

289 synchronization of these neurons to the stimulus frequency (16-21), yet we found that lactate and

290 glutamate increases during PF, not UF. These findings indicate that the stimulation-induced effect

291 on metabolite concentrations is happening downstream the input stage in layer IV, and specifically

292 during the intracortical processing involving output layers II/III. This conclusion is supported by

293 the fact that high-frequency flickering $(30-60 \mathrm{~Hz})$ abolished MUA, a measure of neuronal spiking

294 (putatively from layer II/III) (26), but not single neuron recordings from layer IV (16).

295 Using compartmentalized metabolic modeling, we have recently obtained evidence that the above-

296 mentioned outcome can be explained by distinct signaling mechanisms underlying spiking and

297 synaptic activity (e.g., pre- and postsynaptic ionic currents) that are indeed frequency-dependent

$298(30,51)$. We previously reported that chromatic and achromatic flickering at the same temporal

299 frequency elicited the same neurochemical response in V1 despite differences in the responding

300 neuronal populations (i.e., blob versus interblob) (10). Together with the results that we report

301 here, these observations suggest that lactate and glutamate, and hence aerobic glycolysis, are both 
sensitive to cortical processing (e.g., input/output or excitation/inhibition balance) rather than the targeted neuronal population. Our results can be interpreted in keeping with the concept that

304 increased glutamate levels reflect an upregulation of the malate-aspartate shuttle (MAS) (52),

305 although we were unable to report significant differences in aspartate levels likely due to

306 insufficient sensitivity of MRS at 3T. Notably, MAS does not necessarily correlate with

307 mitochondrial respiration and cerebral blood flow, as evidenced by the findings that (i) oxidative

308 metabolism and $\mathrm{CMRO}_{2}$ are enhanced at both low and high flickering frequencies (26), and (ii)

309 glutamate and aspartate, but not lactate, correlate with BOLD signals during low-frequency $(\sim 8$

$310 \mathrm{~Hz}$ ) visual stimulation $(10,53)$. To what extent aerobic glycolysis and MAS can dissociate from

311 oxidative phosphorylation in the brain remains to be established, but previously published data

312 strongly indicates that the increase in lactate and glutamate levels is the consequence of the surge

313 in glycolysis and the ensuing accumulation of NADH taking place during neuronal activation (54).

314 While the similar BOLD response in V1 is a direct consequence of our experimental design, we

315 additionally found that secondary visual cortices were recruited to a larger extent during PF

316 stimulus than UF stimulus, supporting the argument that their activation is necessary for perception

317 (55-57). The preferential response of secondary visual areas to perceived stimuli that we observe

318 here broadly confirms previous results of a strong BOLD activity in ventral ("visual-for-

319 perception" processing) and dorsal ("visual-for-action" processing) streams during visible stimuli

320 and a reduction of their activity in conditions of invisible stimulation (58).

321 The role of primary visual cortex in stimulus perception is an intense area of research (59-63).

322 Previous fMRI studies investigating the dependence of V1 BOLD activity on visual perception

323 produced controversial results, which might be related to the difficulty of disentangling perception

324 from spatial attention. Indeed, it has been shown that attention, but not perception, modulates the 
BOLD signal in human V1 (32). In fact, perception was found to enhance the BOLD response

326 within V1 for specific experimental designs (e.g., flash suppression of perception) (64). Our study

327 employed a substantially different stimulation paradigm that specifically allowed us, by adjusting

328 the stimulation contrast, to induce the same BOLD response, and inductively an equivalent mean

329 degree of synaptic activity, within V1 under the two experimental conditions (47). Most

330 importantly, we did not use any additional stimulus (e.g., visual masking) to suppress or modulate

331 visual perception. On the contrary, we used a simple way to modulate perception for extended

332 periods of time (required by $1 \mathrm{H}-\mathrm{fMRS}$ ) while maintaining attention at a nearly constant level, as

333 evidenced by pupillometry and task performance. In particular, we employed the well-known

334 dependence of BOLD response in V1 to flickering, which at full-contrast peaks at 4-8 $\mathrm{Hz}$ and

335 settles around $70 \%$ of its maximal value even at frequencies above $30 \mathrm{~Hz}(65)$, i.e., in the absence

336 of flickering perception. Previous literature reported that the peak activations in V1 and secondary

337 visual areas are stimulation-dependent and occur at distinct temporal frequencies $(4 \mathrm{~Hz}$ or $8 \mathrm{~Hz}$ in

$338 \mathrm{~V} 1$, and $20 \mathrm{~Hz}$ or $40 \mathrm{~Hz}$ in secondary visual areas for chromatic or luminance flickering,

339 respectively) (66-70). Interestingly, the preferred frequency of secondary visual areas is just below

340 the $\mathrm{CFF}$ for chromatic flickering $(\sim 25 \mathrm{~Hz})$ and luminance flickering $(\sim 50 \mathrm{~Hz})(71)$. In our

341 experiments, the isoluminant chromatic flickering stimulation at $30 \mathrm{~Hz}$ is above the CFF and

342 accordingly we found that the BOLD response in secondary visual areas drops substantially

343 compared with the concurrent BOLD response in V1. Overall, by using visual stimulations below

344 and above the CFF and adjusting the contrast of the low-frequency stimulus we were able to

345 modulate perception alongside BOLD activity in secondary visual areas with an unchanged BOLD

346 activity in V1.

347 Our study has some limitations. For instance, the fMRI measurements have been obtained using 34830 s epochs, while the fMRS measurements have been obtained using 4 min epochs. Long 
acquisition times are required for achieving a good sensitivity of the 1H-fMRS measurements and

350 a reliable metabolite quantification, even at magnetic fields of 3T. Nonetheless, results in both

351 humans (48) and rats (72) have showed that prolonged (i.e., minutes) stimulations elicit a persistent

352 BOLD response with minimal attenuation. Although we cannot exclude a certain degree of 353 neuronal adaptation, we confirmed that the BOLD effect during the fMRS acquisition was indeed

354 relatively stable as determined by the linewidth changes of $\mathrm{tCr}$ and tNAA signals. Another

355 limitation to consider is represented by the large volume used for $1 \mathrm{H}-\mathrm{fMRS}$ measurements. It could

356 be possible that the observed changes in lactate levels include distinct neuronal populations

357 showing non uniform responses. Indeed, although the main differential effect of frequency was

358 located far from the calcarine sulcus, it also involved some mid hemispheric areas presumably

359 included in the spectroscopic voxel. As an illustration, heterogeneity in the response to stimulation

360 within V1 might be due to, e.g., eccentricity (67), which we however minimized by using a $3^{\circ}$

361 foveal stimulation. There is also an hemispherical lateralization for the spatial frequencies (73),

362 but this is not likely to apply to our study, as we used the same radial checkerboard (i.e., including

363 many spatial frequencies) for both PF and UF. The finding that the changes in BOLD signal during

364 PF and UF were nearly double in the anatomical (i.e., Brodmann atlas-based) V1 compared with

365 the spectroscopic VOI indicates a substantial partial-volume effect. The associated loss of

366 sensitivity entails that the lactate concentration change that we report here might in fact be under-

367 estimated. Finally, we cannot exclude that feedback input to V1 from secondary visual areas might

368 be taking place during stimulus perception (74). However, under our experimental design we were

369 able to fully control BOLD response in V1 by only acting on stimulus contrast, without altering

370 the subjective perception of the stimuli, which indicates that the BOLD activity in V1 largely

371 reflected stimulus features. 
372 In conclusion, in this study, we report for the first time that visual perception has a measurable

373 metabolic effect on V1. Our findings imply that BOLD fMRI and 1H-fMRS are complementary

374 techniques, capable of highlight different aspects of neural activation and stimulus processing, e.g.,

375 under conditions in which synaptic and spiking activity are partially disentangled due to an altered

376 balance between excitation and inhibition.

377 Although we focused on the visual pathways, it is conceivable that our results can be translated to 378 other sensory modalities. For example, tactile $(75)$ or auditory $(76,77)$ stimulations during

379 vegetative state can still activate primary somatosensory cortex (S1) or primary auditory cortex

380 (A1), respectively, in the absence of perception and without the involvement of higher-order 381 associative areas.

382 We suggest that the cortical metabolic profile might be an indicator of sensory perception, in

383 keeping with the dynamics of lactate and glutamate across the sleep-wake cycle $(31,40,78,79)$.

384 In particular, elevated brain lactate and glutamate levels are associated with wakefulness and 385 memory formation, which naturally require the processing of incoming sensory stimuli, like the 386 control exerted by the central visual pathways for either gating or filtering out behaviorally relevant 387 or irrelevant visual information. In particular, aerobic glycolysis and lactate might reflect cortical 388 information processing and, in turn, intracortical communication, in agreement with the relation 389 between regional metabolic rates of glucose utilization and resting-state network dynamics in the 390 cerebral cortex (80-84). 


\section{Materials and methods}

\section{Setup}

394 MR measurements were performed on a 3T head-only scanner (Siemens Allegra, Erlangen,

395 Germany), equipped with high performance gradients (amplitude $40 \mathrm{mT} / \mathrm{m}$, rise time $100 \mu \mathrm{s}$ ). A

396 custom-built quadrature surface coil (loop and butterfly design, Rapid Biomedical, Rimpar,

397 Germany) was used for both RF transmission and detection. The coil design traded usable

398 volume (see Figure 2 - figure supplement 1) for peak sensitivity. For imaging, first and second

399 order shim terms were adjusted using the automatized Siemens routine based on field map

400 acquisition and fitting. For MRS, shimming was optimized using FASTMAP with EPI readout

401 (85) and manually refined when necessary to reach a water linewidth of less than $9 \mathrm{~Hz}$.

\section{Subjects}

403 A total of 32 healthy volunteers were initially enrolled for this study after they gave informed 404 consent, according to the Helsinki declaration and to European Union regulations, and following 405 the approval by the Ethics Committee of Fondazione Santa Lucia (Rome). Exclusion criteria 406 included any kind of previous neurological or psychiatric disease and impaired visual acuity.

407 Thirteen subjects were discarded either upon online fMRI processing (see below) or in early 408 quality control on data, because of motion (3) or suboptimal anatomical features, with the most 409 activated area in an unfavourable position respect to the coil sensitive volume (10). Nineteen 410 healthy volunteers ( 10 females, 9 males; age $25 \pm 4$ years, mean $\pm \mathrm{SD}$; age range 20 to 35 years) were

411 thus considered for this study. Sample size calculations performed before the study assumed a two-

412 tail paired t-test design, a power of 0.95 and were based on an effect magnitude for lactate change 413 (photic stimulation vs rest) of $0.20 \pm 0.15 \mu \mathrm{mol} / \mathrm{g}$ as estimated in our previous works at 7T (10).

414 Reduced sensitivity of 3 vs 7T was epmpirically accounted for by larger VOI and extended data 
415 averaging (144 transients per condition, $10 \mathrm{ml} \mathrm{VOI}$ at 3T vs 64 transients per condition, $8 \mathrm{ml}$ VOI

416 at 7T). The resulting required sample size of 10 was roughly doubled to account for multiple

417 comparisons and other suboptimal procedures at 3T compared to 7T.

418 Visual stimulation

419 Visual stimulation consisted in a radial checkerboard flickering either at $7.5 \mathrm{~Hz}$ (perceived

420 flickering stimulation, PF) or at $30 \mathrm{~Hz}$ (unperceived flickering stimulation, UF). The alternating

421 frames included either a gray-green or a gray-red checkerboard. The stimuli were programmed in

422 Cogent 2000 version 1.29 working under Matlab 2006b (The Mathworks, Natick, MA, USA). and

423 delivered using an MR-compatible fMRI system with stereo 3D goggles (VisuaStim Digital,

424 Resonance Technology, Inc., Northridge, California, USA). Subjects were fitted with the

425 VisuaStim video goggles (Resolution: SXGA 1280x1024 pixels, Refresh Rate: 60Hz, Field of

426 View: 30 degrees Horizontal x 24 degrees Vertical, White Luminance: $70 \mathrm{~cd} / \mathrm{m} 2 \mathrm{max}$, Contrast

427 Ratio: intrinsic 100:1 measured per VESA FPDM Standard).

\section{Experimental protocol}

429 Visual stimulations were presented in eight (fMRI) or four (fMRS) epochs, with stimulation

430 epochs (either PF or UF) interleaved by rest (isoluminant, uniform grey images) epochs. Since the

431 CFF for loss of perception is higher for luminance than for chromatic flickering (25), before each

432 session the brightness of green squares during the UF condition (i.e. isoluminant condition) was

433 adjusted interactively by the subject, who piloted increasing and decreasing brightness ramps and

434 was instructed to identify the brighness levels corresponding to loss of luminance flickering

435 perception of the resulting yellow. The green level was then set midway between the two

436 perceptual vanishing levels. The stimulus contrast was adjusted in preliminary acquisitions on five

437 subjects, in order to induce a comparable BOLD response to PF and UF in V1. During the initial 
fMRI sessions epochs lasted 30 seconds each (total 4 minutes), while for fMRS acquisitions epochs were 4 minutes long (total 16 minutes). Each subject underwent $1 \mathrm{fMRI}$ and $2 \mathrm{fMRS}$ runs (10 minutes apart, without moving the subject from inside the scanner); the order of PF and UF

441 conditions was counterbalanced within each subject, and the initial stimulation type was 442 randomized between subjects.

\section{Task}

444 In order to maintain visual fixation and keep the attentional state constant, the subjects were asked

445 to focus on a central target (a cross) and to press a button whenever the target rotated. Subjects

446 were specifically instructed to maintain their attention on the fixation cross rather than focusing

447 on reaction times (i.e., speed to push the button). The number of rotations was constant across the

448 different epochs (3 for each fMRI epoch and 24 for each 1H-fMRS epoch, or approx. 1 rotation

449 every 10 seconds), while the exact timing of the rotation was pseudo-randomized (range 2-18

450 seconds).

\section{Anatomical and fMRI data acquisition}

452 Each study started with an anatomical acquisition (MPRAGE T1-weighted volumetric scan, 453 resolution $1.2 \times 1.2 \times 1.2 \mathrm{~mm}^{3}$, para--axial slices, in-plane FOV $190 \times 70 \mathrm{~mm}^{2}$, TE=4.38 ms,

$454 \mathrm{TR}=2000 \mathrm{~ms}, \mathrm{TI}=910 \mathrm{~ms}, \mathrm{FA}=8^{\circ}$ ). Then, one fMRI session (pseudo-randomized order of 455 stimulation across subjects) was acquired (gradient echo with EPI readout, resolution $2.2 \times 2.2 \times 2.2$ $456 \mathrm{~mm}^{3}, 26$ para--axial contiguous slices, FOV $\left.190 \times 70 \mathrm{~mm}^{2}, \mathrm{TE}=30 \mathrm{~ms}, \mathrm{TR}=1500 \mathrm{~ms}, \mathrm{FA}=70^{\circ}\right)$.

457 fMRI scans were processed online for subsequent MRS voxel positioning (online processing 458 included motion correction, smoothing, cross-correlation with a square-wave model; the two scans 459 following each condition change were discarded in order to reduce the effects of BOLD signal 460 transients. Online processign was discarded after voxel positioning). 
461 To confirm the absence of any detectable brain pathology in our subjects, T1 and T2 weighted

462 anatomical scans were acquired with a standard volume birdcage coil after the end of the functional

463 scans. Anatomical scans included an MPRAGE acquisition (resolution $1.0 \times 1.0 \times 1.0 \mathrm{~mm}^{3}$, para-

464 axial slices, in-plane FOV $256 \times 160 \mathrm{~mm}^{2}, \mathrm{TE}=2.48 \mathrm{~ms}, \mathrm{TR}=2150 \mathrm{~ms}, \mathrm{TI}=1000 \mathrm{~ms}, \mathrm{FA}=8^{\circ}$ ), that

465 was later used during the post-processing for normalization purposes.

fMRS data acquisition

467 The spectroscopic voxel (size $25 \times 20 \times 20 \mathrm{~mm}^{3}$ ) was localized in the most activated area within V1,

468 based on both anatomical scan and results of the online fMRI processing. The voxel was located

469 either left or right of the interemispheric fissure to minimize the cerebrospnial fluid fraction in the

470 VOI. Two MRS sessions were acquired with an optimized, in-house written STEAM sequence

$471\left(\mathrm{TE}=7 \mathrm{~ms}, \mathrm{TM}=50 \mathrm{~ms}, \mathrm{TR}=3000 \mathrm{~ms}, \mathrm{FA}=70^{\circ}\right)$ which included outer volume saturation and

472 VAPOR water suppression $(86,87)$. An eight-step phase cycle was used; transients were averaged

473 within each phase cycle, and each phase cycle was saved separately for further processing. Water

474 unsuppressed data were acquired from the same voxel for eddy currents compensation (88). In

475 order to minimize T1 weighting, the flip angle was kept below the calculated Ernst angle in both

476 fMRI and fMRS acquisitions.

\section{Pupillometry}

478 In order to monitor attentional state with a physiological parameter, we acquired pupillometry data

479 using an eye-tracking system (Applied Science Laboratories, model 504) equipped with remote

480 pan/tilt optic infrared module and a video camera that was custom-adapted for use in the scanner.

481 Subject gaze position and pupil size data were processed as previously described (33). 


\section{fMRI data processing}

483 fMRI (offline) processing was performed with routines from SPM12 (Wellcome Trust Centre for

484 Neuroimaging, UCL) working under Matlab 2018b, AFNI (89) and FSL5 (90), and with custom

485 Matlab routines. For volume-of-interest (VOI) based analysis, fMRI data were realigned to their

486 mean image. For voxel-based analysis, data were also normalized to the MNI template by using

487 the non linear transform calculated on the MPRAGE acquired with the volume coil, after a linear

488 co-registration that used the surface coil MPRAGE image as intermediate step to best match the

489 volume coil MPRAGE to the fMRI series. A Gaussian smoothing with kernel $4 \times 4 \times 4 \mathrm{~mm}^{3}$ was

490 then applied before fitting to a model that included the hemodynamic response. Only for

491 visualization purposes, MR images were assembled with standard image processing tools, without

492 any kind of adjustment. Head motion during fMRI acquisitions was evaluated using the framewise

493 displacement, which was calculated as the L1-norm of the realignment-derived parameters after

494 converting angles to linear displacements (91).

\section{5 fMRS data processing}

496 MRS data were preprocessed using jMRUI 5.2 (92) and custom Matlab routines. Data were

497 corrected for residual eddy currents, individually phased and frequency shifted to compensate $\mathrm{B}_{0}$

498 drifts, and averaged in blocks corresponding to each rest or stimulation epoch. The first 8 transients

499 of each epoch, i.e. the first full phase cycle (24 s) were discarded to avoid metabolic transients (3).

500 Subsequent phase cyles were inspected individually. They consistently showed good water

501 suppression and no trace of lipidic contamination. A few 8-transient spectra (maximum one in each

502 epoch) featured anomalous line broadening, line splitting or otherwise reduced quality, putatively

503 related to subject motion or deep inspiration, and were discarded before averaging. Each epoch

504 spectrum was thus the average of 64-72 transients. The resulting averages were finally quantified 
using LCModel 6.3-1 (93) with a tailored basis set. Basis metabolites included alanine, aspartate

506 (Asp), creatine (Cr), $\gamma$-Aminobutyric acid (GABA), glucose, glutamine (Gln), glutamate (Glu),

507 glycine, glycerylphosphorylcholine, glutathione (GSH), lactate (Lac), myo-inositol (Ins), $N$ -

508 acetylaspartate (NAA), $N$-acetylaspartylglutamate (NAAG), phosphocholine, phosphocreatine,

509 phosphorylethanolamine, scyllo-inositol, and taurine (Tau). Glucose, an important marker of

510 energy metabolism, whose changes have also been reported in previous 7 T studies $(3,9)$, was not

511 included in the basis set due to highly unreliable quantification observed in preliminary tests.

512 Metabolite spectra were simulated using GAVA (94), including information on the sequence pulse

513 program. The basis set included also a subject-specific macromolecular (MM) signal, that was

514 acquired on each subject in the occipital region, using a double inversion recovery approach

515 (STEAM, TI1=1700 ms, TI2=520 ms, TE=7 ms, TM=50 ms, TR=2000 ms, FA=90') (95), that

516 resulted in almost complete metabolite nulling, averaged between subjects, and then modeled with

517 Hankel-Lanczos singular value decomposition. LCmodel quantifications with Cramér-Rao lower

518 bounds (CRLB) above 30\% were discarded, except for Lac for which the threshold was set at 35\%.

519 Since this study is focused on epoch to epoch metabolic changes, absolute quantification with

520 water referencing was not performed to avoid the associated uncertainty. Metabolites were rather

521 referred to the internal creatine signal, assumed to be $7.5 \mu \mathrm{mol} / \mathrm{g}$ in the VOI. Finally, concentrations

522 measured in homologous epochs were averaged, obtaining for each subject four concentrations,

523 corresponding to two stimulation conditions and to the relevant rest reference (the resting epoch

524 immediately successive to a condition). Eleven metabolites were quantified in at least 15 subjects

525 (80\% of participants). These included aspartate, total creatine, GABA, glutamate, glutamine,

526 glutathione, lactate, myo-inositol, N-acetylaspartate, total choline, taurine. In order to exclude

527 BOLD adaptation during the 4-min duration of the fMRS epochs, we determined the BOLD effect

528 during fMRS scanning as the kernel size (in $\mathrm{Hz}$ ) that minimized the amplitude of the difference 
529 spectra between stimulated epochs (either PF and UF) and the preceding resting epoch. All spectra

530 were then averaged according to three categories: rest, PF and UF conditions, and spectral

531 differences were calculated between conditions.

\section{Statistics}

533 For pupillometry and task performance results, statistical comparisons were made using Student's

534 t-test and One-Way ANOVA on the rest, UF, and PF conditions. No post-hoc test was necessary.

535 For fMRI results, correction for multiple comparisons in functional voxel-based analysis was

536 performed using False Discovery Rate (FDR) correction. Resulting clusters were also checked

537 through Monte Carlo Simulation using the AFNI tool Alphasim (89) after estimation of residuals

538 smoothness.

539 For fMRS results, statistical analysis was restricted to those reliably quantified metabolites

540 associated with energy metabolism that showed consistent funcional changes in previous fMRS

541 studies $(4,9,10)$, namely Lac, Glu, and Asp. Metabolite concentration changes referred to the

542 corresponding resting epoch and between different active conditions were tested using paired two-

543 sample t-tests, with FDR correction for 9 multiple comparisons.

544 Data were presented as the mean \pm standard deviation (SD). A p-value, or a qFDR-value where 545 relevant, of less than 0.05 was considered as statistically significant. 


\section{References}

1. Siesjo DP (1978) Brain Energy Metabolism (Wiley, New York).

2. Mangia S, et al. (2009) Metabolic and hemodynamic events after changes in neuronal activity: current hypotheses, theoretical predictions and in vivo NMR experimental findings. $J$ Cereb Blood Flow Metab 29(3):441-463.

3. Mangia S, et al. (2007) Sustained neuronal activation raises oxidative metabolism to a new steady-state level: evidence from $1 \mathrm{H}$ NMR spectroscopy in the human visual cortex. J Cereb Blood Flow Metab 27(5):1055-1063.

4. Lin Y, Stephenson MC, Xin L, Napolitano A, \& Morris PG (2012) Investigating the metabolic changes due to visual stimulation using functional proton magnetic resonance spectroscopy at $7 \mathrm{~T}$. J Cereb Blood Flow Metab 32(8):1484-1495.

5. Boillat Y, Xin L, van der Zwaag W, \& Gruetter R (2020) Metabolite concentration changes associated with positive and negative BOLD responses in the human visual cortex: A functional MRS study at 7 Tesla. $J$ Cereb Blood Flow Metab 40(3):488-500.

6. Fernandes CC, Lanz B, Chen C, \& Morris PG (2020) Measurement of brain lactate during visual stimulation using a long TE semi-LASER sequence at 7 T. NMR in biomedicine 33(4):e4223.

7. Schaller B, Mekle R, Xin L, Kunz N, \& Gruetter R (2013) Net increase of lactate and glutamate concentration in activated human visual cortex detected with magnetic resonance spectroscopy at 7 tesla. Journal of neuroscience research 91(8):1076-1083.

8. Schaller B, Xin L, O'Brien K, Magill AW, \& Gruetter R (2014) Are glutamate and lactate increases ubiquitous to physiological activation? A (1)H functional MR spectroscopy study during motor activation in human brain at 7Tesla. Neurolmage $93 \mathrm{Pt}$ 1:138-145.

9. Bednarik $\mathrm{P}$, et al. (2015) Neurochemical and BOLD responses during neuronal activation measured in the human visual cortex at 7 Tesla. J Cereb Blood Flow Metab 35(4):601-610.

10. Bednarik $\mathrm{P}$, et al. (2018) Neurochemical responses to chromatic and achromatic stimuli in the human visual cortex. J Cereb Blood Flow Metab 38(2):347-359.

11. Lin AL, Fox PT, Hardies J, Duong TQ, \& Gao JH (2010) Nonlinear coupling between cerebral blood flow, oxygen consumption, and ATP production in human visual cortex. Proceedings of the National Academy of Sciences of the United States of America 107(18):8446-8451.

12. Dienel GA (2019) Brain Glucose Metabolism: Integration of Energetics with Function. Physiological reviews 99(1):949-1045.

13. D'Souza RD \& Burkhalter A (2017) A Laminar Organization for Selective Cortico-Cortical Communication. Front Neuroanat 11:71-71.

14. Crick F \& Koch C (1995) Are we aware of neural activity in primary visual cortex? Nature 375(6527):121123.

15. Lauritzen M (2001) Relationship of spikes, synaptic activity, and local changes of cerebral blood flow. $J$ Cereb Blood Flow Metab 21(12):1367-1383.

16. Gur M \& Snodderly DM (1997) A dissociation between brain activity and perception: chromatically opponent cortical neurons signal chromatic flicker that is not perceived. Vision Res 37(4):377-382.

17. Regan D (1989) Human brain electrophysiology: evoked potentials and evoked magnetic fields in science and medicine. (Elsevier, New York).

18. Herrmann CS (2001) Human EEG responses to 1-100 Hz flicker: resonance phenomena in visual cortex and their potential correlation to cognitive phenomena. Experimental brain research 137(3-4):346-353.

19. Pastor MA, Artieda J, Arbizu J, Valencia M, \& Masdeu JC (2003) Human cerebral activation during steadystate visual-evoked responses. The Journal of neuroscience : the official journal of the Society for Neuroscience 23(37):11621-11627.

20. Norcia AM, Appelbaum LG, Ales JM, Cottereau BR, \& Rossion B (2015) The steady-state visual evoked potential in vision research: A review. Journal of vision 15(6):4.

21. Vialatte FB, Maurice M, Dauwels J, \& Cichocki A (2010) Steady-state visually evoked potentials: focus on essential paradigms and future perspectives. Progress in neurobiology 90(4):418-438.

22. Sachidhanandam S, Sreenivasan V, Kyriakatos A, Kremer Y, \& Petersen CC (2013) Membrane potential correlates of sensory perception in mouse barrel cortex. Nature neuroscience 16(11):1671-1677.

23. Alais D, Locke SM, Leung J, \& Van der Burg E (2016) No attentional capture from invisible flicker. Scientific Reports 6(1):29296.

24. Lorenceau J (1987) Recovery from contrast adaptation: effects of spatial and temporal frequency. Vision Res 27(12):2185-2191.

25. Jiang Y, Zhou K, \& He S (2007) Human visual cortex responds to invisible chromatic flicker. Nature neuroscience 10(5):657-662.

26. Viswanathan A \& Freeman RD (2007) Neurometabolic coupling in cerebral cortex reflects synaptic more than spiking activity. Nat. Neurosci. 10(10):1308--1312.

27. Logothetis NK, Pauls J, Augath M, Trinath T, \& Oeltermann A (2001) Neurophysiological investigation of the basis of the fMRI signal. Nature 412(6843):150-157. 
bioRxiv preprint doi: https://doi.org/10.1101/2021.09.17.460759; this version posted September 20, 2021. The copyright holder for this preprint (which was not certified by peer review) is the author/funder, who has granted bioRxiv a license to display the preprint in perpetuity. It is made available under aCC-BY 4.0 International license.

28. Hecht S \& Shlaer S (1936) INTERMITTENT STIMULATION BY LIGHT : V. THE RELATION BETWEEN INTENSITY AND CRITICAL FREQUENCY FOR DIFFERENT PARTS OF THE SPECTRUM. The Journal of general physiology 19(6):965-977.

29. DiNuzzo M, Maraviglia B, \& Giove F (2011) Why does the brain (not) have glycogen? Bioessays 33(5):319326.

30. DiNuzzo M \& Giove F (2012) Activity-dependent energy budget for neocortical signaling: effect of short-term synaptic plasticity on the energy expended by spiking and synaptic activity. Journal of neuroscience research 90(11):2094-2102.

31. DiNuzzo M \& Nedergaard M (2017) Brain energetics during the sleep-wake cycle. Curr Opin Neurobiol 47:65-72.

32. Watanabe M, et al. (2011) Attention but not awareness modulates the BOLD signal in the human V1 during binocular suppression. Science 334(6057):829-831.

33. DiNuzzo M, et al. (2019) Brain Networks Underlying Eye's Pupil Dynamics. Frontiers in neuroscience 13:965.

34. Gelbard-Sagiv H, Magidov E, Sharon H, Hendler T, \& Nir Y (2018) Noradrenaline Modulates Visual Perception and Late Visually Evoked Activity. Current biology : CB 28(14):2239-2249.e2236.

35. Eickhoff SB, et al. (2005) A new SPM toolbox for combining probabilistic cytoarchitectonic maps and functional imaging data. Neurolmage 25(4):1325-1335.

36. Zhu XH \& Chen W (2001) Observed BOLD effects on cerebral metabolite resonances in human visual cortex during visual stimulation: a functional (1)H MRS study at $4 \mathrm{~T}$. Magnetic resonance in medicine 46(5):841-847.

37. Herculano-Houzel S (2011) Scaling of brain metabolism with a fixed energy budget per neuron: implications for neuronal activity, plasticity and evolution. PloS one 6(3):e17514.

38. Niven JE \& Laughlin SB (2008) Energy limitation as a selective pressure on the evolution of sensory systems. The Journal of experimental biology 211(Pt 11):1792-1804.

39. DiNuzzo M (2016) Astrocyte-Neuron Interactions during Learning May Occur by Lactate Signaling Rather than Metabolism. Frontiers in integrative neuroscience 10:2.

40. Aalling NN, Nedergaard M, \& DiNuzzo M (2018) Cerebral Metabolic Changes During Sleep. Current neurology and neuroscience reports 18(9):57.

41. Newman LA, Korol DL, \& Gold PE (2011) Lactate produced by glycogenolysis in astrocytes regulates memory processing. PloS one 6(12):e28427.

42. Scavuzzo CJ, Rakotovao I, \& Dickson CT (2020) Differential effects of L- and D-lactate on memory encoding and consolidation: Potential role of HCAR1 signaling. Neurobiology of Learning and Memory 168:107151.

43. Descalzi G, Gao V, Steinman MQ, Suzuki A, \& Alberini CM (2019) Lactate from astrocytes fuels learninginduced mRNA translation in excitatory and inhibitory neurons. Communications Biology 2(1):247.

44. Suzuki A, et al. (2011) Astrocyte-neuron lactate transport is required for long-term memory formation. Cell 144(5):810-823.

45. Dienel GA (2019) Does shuttling of glycogen-derived lactate from astrocytes to neurons take place during neurotransmission and memory consolidation? Journal of neuroscience research.

46. Steinman MQ, Gao V, \& Alberini CM (2016) The Role of Lactate-Mediated Metabolic Coupling between Astrocytes and Neurons in Long-Term Memory Formation. Frontiers in integrative neuroscience 10:10-10.

47. Logothetis NK (2008) What we can do and what we cannot do with fMRI. Nature 453(7197):869-878.

48. Mangia S, et al. (2007) Dynamics of lactate concentration and blood oxygen level-dependent effect in the human visual cortex during repeated identical stimuli. Journal of neuroscience research 85(15):3340-3346.

49. Peca S, et al. (2010) Metabolic correlatives of brain activity in a FOS epilepsy patient. NMR in biomedicine 23(2):170-178.

50. Buxton RB, Griffeth VE, Simon AB, Moradi F, \& Shmuel A (2014) Variability of the coupling of blood flow and oxygen metabolism responses in the brain: a problem for interpreting BOLD studies but potentially a new window on the underlying neural activity. Frontiers in neuroscience 8:139.

51. DiNuzzo M, Mangia S, Maraviglia B, \& Giove F (2014) Effect of short-term synaptic plasticity on the relationship between neuronal activity, BOLD, CMRO2 and CMRGlc studied by metabolic modeling of neuron-glia interaction. Proc Intl Soc Magn Reson Med 22:1884.

52. Mangia S, Giove F, \& DiNuzzo M (2012) Metabolic pathways and activity-dependent modulation of glutamate concentration in the human brain. Neurochemical research 37(11):2554-2561.

53. Ip IB, et al. (2017) Combined fMRI-MRS acquires simultaneous glutamate and BOLD-fMRI signals in the human brain. Neurolmage 155:113-119.

54. Diaz-Garcia CM, et al. (2017) Neuronal Stimulation Triggers Neuronal Glycolysis and Not Lactate Uptake. Cell metabolism 26(2):361-374.e364.

55. Salminen-Vaparanta N, Koivisto M, Vorobyev V, Alakurtti K, \& Revonsuo A (2019) Does TMS on V3 block conscious visual perception? Neuropsychologia 128:223-231.

56. Tong F (2003) Primary visual cortex and visual awareness. Nature reviews. Neuroscience 4(3):219-229.

57. Lamme VAF, Supèr H, Landman R, Roelfsema PR, \& Spekreijse H (2000) The role of primary visual cortex (V1) in visual awareness. Vision Research 40(10):1507-1521.

58. Hesselmann $G$ \& Malach $R$ (2011) The link between fMRI-BOLD activation and perceptual awareness is "stream-invariant" in the human visual system. Cerebral cortex (New York, N.Y. : 1991) 21(12):2829-2837. 
59. Polonsky A, Blake R, Braun J, \& Heeger DJ (2000) Neuronal activity in human primary visual cortex correlates with perception during binocular rivalry. Nature neuroscience 3(11):1153-1159.

60. Wunderlich K, Schneider KA, \& Kastner S (2005) Neural correlates of binocular rivalry in the human lateral geniculate nucleus. Nature neuroscience 8(11):1595-1602.

61. Leopold DA \& Logothetis NK (1996) Activity changes in early visual cortex reflect monkeys' percepts during binocular rivalry. Nature 379(6565):549-553.

62. Gail A, Brinksmeyer HJ, \& Eckhorn R (2004) Perception-related modulations of local field potential power and coherence in primary visual cortex of awake monkey during binocular rivalry. Cerebral cortex (New York, N.Y. : 1991) 14(3):300-313.

63. Maier A, et al. (2008) Divergence of $\mathrm{FMR}$ and neural signals in $\mathrm{V} 1$ during perceptual suppression in the awake monkey. Nature neuroscience 11(10):1193-1200.

64. Yuval-Greenberg S \& Heeger DJ (2013) Continuous flash suppression modulates cortical activity in early visual cortex. The Journal of neuroscience : the official journal of the Society for Neuroscience 33(23):96359643.

65. Thomas CG \& Menon RS (1998) Amplitude response and stimulus presentation frequency response of human primary visual cortex using BOLD EPI at 4 T. Magnetic resonance in medicine 40(2):203-209.

66. Kastner S, et al. (2004) Functional imaging of the human lateral geniculate nucleus and pulvinar. Journal of neurophysiology 91(1):438-448.

67. Chai $\mathrm{Y}$, et al. (2019) Visual temporal frequency preference shows a distinct cortical architecture using fMRI. Neurolmage 197:13-23.

68. Fox PT, et al. (1986) Mapping human visual cortex with positron emission tomography. Nature 323(6091):806-809.

69. Kwong KK, et al. (1992) Dynamic magnetic resonance imaging of human brain activity during primary sensory stimulation. Proceedings of the National Academy of Sciences of the United States of America 89(12):5675-5679.

70. Singh KD, Smith AT, \& Greenlee MW (2000) Spatiotemporal frequency and direction sensitivities of human visual areas measured using fMRI. Neurolmage 12(5):550-564.

71. Shady S, MacLeod DIA, \& Fisher HS (2004) Adaptation from invisible flicker. Proc. Natl. Acad. Sci. U. S. A. 101(14):5170--5173.

72. Just N, Xin L, Frenkel H, \& Gruetter R (2013) Characterization of sustained BOLD activation in the rat barrel cortex and neurochemical consequences. Neurolmage 74:343-351.

73. Kauffmann L, Ramanoël S, \& Peyrin C (2014) The neural bases of spatial frequency processing during scene perception. Frontiers in integrative neuroscience 8(37).

74. Watkins S, Shams L, Tanaka S, Haynes JD, \& Rees G (2006) Sound alters activity in human V1 in association with illusory visual perception. Neurolmage 31(3):1247-1256.

75. Laureys S, et al. (2002) Cortical processing of noxious somatosensory stimuli in the persistent vegetative state. Neurolmage 17(2):732-741.

76. Laureys S, et al. (2000) Auditory processing in the vegetative state. Brain : a journal of neurology 123 ( Pt 8):1589-1601.

77. Boly M, et al. (2004) Auditory processing in severely brain injured patients: differences between the minimally conscious state and the persistent vegetative state. Archives of neurology 61(2):233-238.

78. Naylor E, et al. (2012) Lactate as a biomarker for sleep. Sleep 35(9):1209-1222.

79. Naylor E, et al. (2011) Simultaneous real-time measurement of EEG/EMG and L-glutamate in mice: A biosensor study of neuronal activity during sleep. Journal of electroanalytical chemistry (Lausanne, Switzerland) 656(1-2):106-113.

80. Spetsieris PG, et al. (2015) Metabolic resting-state brain networks in health and disease. Proceedings of the National Academy of Sciences of the United States of America 112(8):2563-2568.

81. Thompson GJ (2018) Neural and metabolic basis of dynamic resting state fMRI. Neurolmage 180(Pt B):448462.

82. Noack R, Manjesh C, Ruszinko M, Siegelmann H, \& Kozma R (2017) Resting state neural networks and energy metabolism. 2017 International Joint Conference on Neural Networks (IJCNN), pp 228-235.

83. Jamadar SD, et al. (2021) Metabolic and Hemodynamic Resting-State Connectivity of the Human Brain: A High-Temporal Resolution Simultaneous BOLD-fMRI and FDG-fPET Multimodality Study. Cerebral Cortex.

84. Su H, et al. (2018) Regional cerebral metabolism alterations affect resting-state functional connectivity in major depressive disorder. Quantitative Imaging in Medicine and Surgery 8(9):910-924.

85. Gruetter R \& Tkác I (2000) Field mapping without reference scan using asymmetric echo-planar techniques. Magnetic resonance in medicine 43(2):319-323.

86. Tkác I, Starcuk Z, Choi IY, \& Gruetter R (1999) In vivo 1H NMR spectroscopy of rat brain at $1 \mathrm{~ms}$ echo time. Magnetic resonance in medicine 41(4):649-656.

87. Tkác I, et al. (2001) In vivo 1H NMR spectroscopy of the human brain at 7 T. Magnetic resonance in medicine 46(3):451-456.

88. Klose U (1990) In vivo proton spectroscopy in presence of eddy currents. Magnetic resonance in medicine 14(1):26-30.

89. Cox RW (1996) AFNI: software for analysis and visualization of functional magnetic resonance neuroimages. Computers and biomedical research, an international journal 29(3):162-173. 
bioRxiv preprint doi: https://doi.org/10.1101/2021.09.17.460759; this version posted September 20, 2021. The copyright holder for this preprint (which was not certified by peer review) is the author/funder, who has granted bioRxiv a license to display the preprint in perpetuity. It is made available under aCC-BY 4.0 International license.

90. Jenkinson M, Beckmann CF, Behrens TE, Woolrich MW, \& Smith SM (2012) Fsl. Neurolmage 62(2):782790.

91. Power JD, Barnes KA, Snyder AZ, Schlaggar BL, \& Petersen SE (2012) Spurious but systematic correlations in functional connectivity MRI networks arise from subject motion. Neurolmage 59(3):21422154.

92. Naressi A, et al. (2001) Java-based graphical user interface for the MRUI quantitation package. Magma (New York, N.Y.) 12(2-3):141-152.

93. Provencher SW (1993) Estimation of metabolite concentrations from localized in vivo proton NMR spectra. Magnetic resonance in medicine 30(6):672-679.

94. Soher BJ, Young K, Bernstein A, Aygula Z, \& Maudsley AA (2007) GAVA: spectral simulation for in vivo MRS applications. Journal of magnetic resonance (San Diego, Calif. : 1997) 185(2):291-299.

95. de Graaf RA, et al. (2006) High magnetic field water and metabolite proton T1 and T2 relaxation in rat brain in vivo. Magnetic resonance in medicine 56(2):386-394. 
Acknowledgments

547 The authors wish to thank Edward J. Auerbach for the FASTMAP implementation on Siemens

548 platform, provided by the University of Minnesota under a C2P agreement, and for his help with

549 the setup of offline shim currents calculation. Siemens Healthineers is acknowledged for providing

550 source code and information on the shim coils.

\section{Data and materials availability}

553 The study was developed using SPM12 (https://www.fil.ion.ucl.ac.uk/spm/software/spm12/),

554 LCmodel (http://s-provencher.com/lcmodel.shtml), jMRUI (http://www.jmrui.eu/) and AFNI

555 (https://afni.nimh.nih.gov/).

556 Data used for all the figures and for Tables 2-3 is available as source data to each element. Source

557 data include also custom Matlab code for processing related to each figure.

558 The raw data include sensitive data. The raw dataset cannot be made available in a public

559 repository because of constraints originally set by the Ethics Committee and included in the

560 informed consent signed by participants. Raw data that support the findings of this study are

561 available from the corresponding author upon signing a MTA that would include:

- A list of authorized researchers.

563 - A commitment to not disclose the raw data to persons not included in the list.

564 - A commitment to destroy the raw data when legitimate use is finished.

565 Commercial use of the raw data is not allowed. 
bioRxiv preprint doi: https://doi.org/10.1101/2021.09.17.460759; this version posted September 20, 2021. The copyright holder for this preprint (which was not certified by peer review) is the author/funder, who has granted bioRxiv a license to display the preprint in perpetuity. It is made available under aCC-BY 4.0 International license.

\section{$567 \quad$ Ethics statement}

568 All experiments with human subjects performed by the authors complied with all applicable ethical

569 standards, including the Helsinki declaration and its amendments, institutional/national research

570 committee standards, and international/national/institutional guidelines. 


\section{Supplementary Materials for}

\section{Perception affects the brain's metabolic response to sensory stimulation}

Mauro DiNuzzo, Silvia Mangia, Marta Moraschi, Daniele Mascali, Gisela E. Hagberg, and Federico Giove*

*Corresponding author. Email: federico.giove@cref.it

\section{This material includes:}

Figure 1 - figure supplement 1-3

Figure 2 - figure supplement 1

Figure 4 - figure supplement 1

Other Supplementary Materials for this manuscript include the following:

\section{Movie S1}

\section{NOTE}

Following eLife style suggestions, Supplementary figures are merged to main text as "Figure supplements" and linked to main figures as indicated by the title of each supplementary figure 


\section{Figure 1 - figure supplement 1}
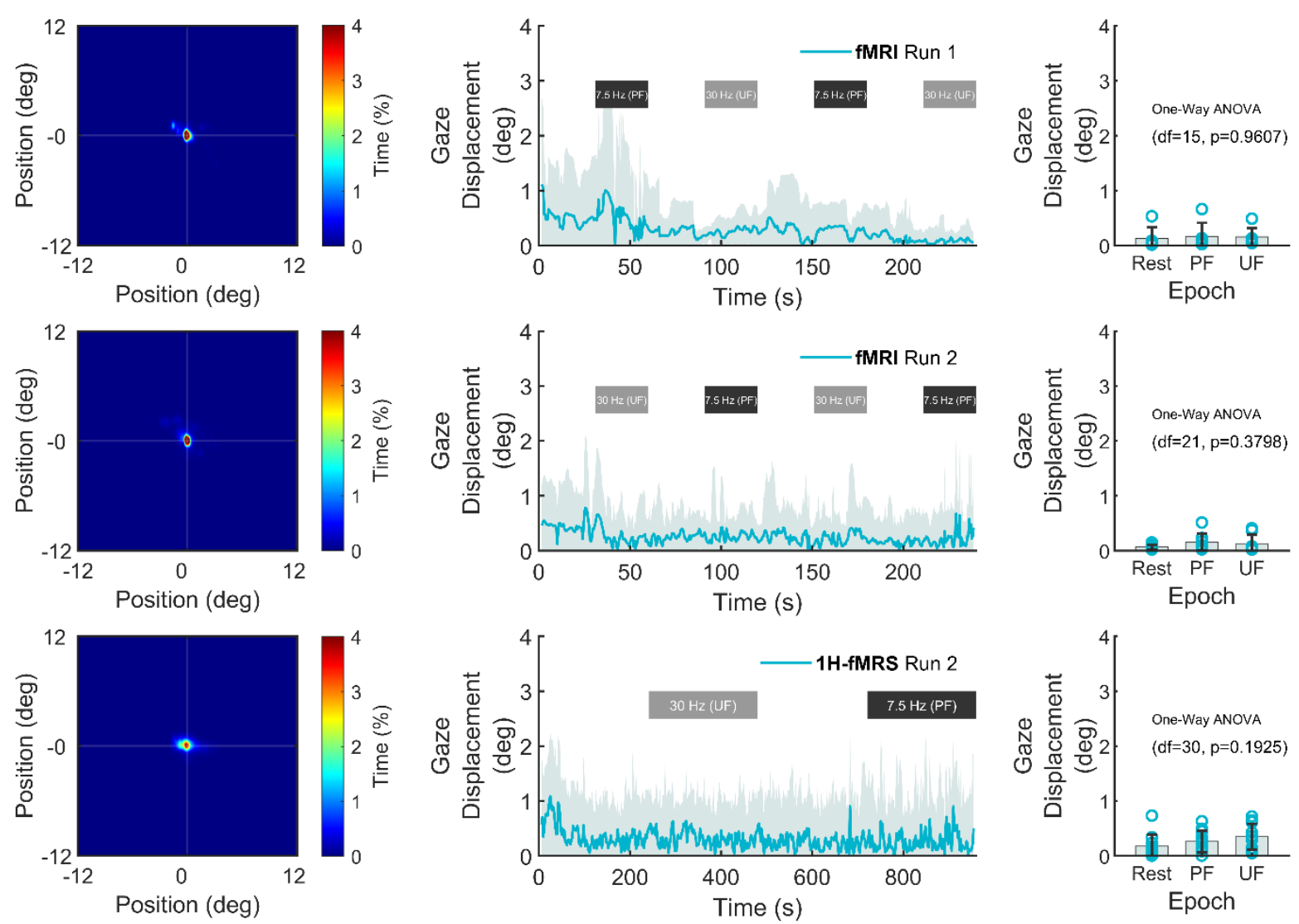

Left: average heatmap of eyes position (across subjects) during the different sessions. (Center) Stability of mean gaze displacement from the fixation point. Right: gaze displacement was not different across conditions (One-Way ANOVA, $\mathrm{p}>0.19$ ). Error bars correspond to SD. Sessions are shown in different rows (Top: fMRI Run 1; Middle: fMRI Run 2; Bottom: 1H-fMRS Run 2). 


\section{Figure 1 - figure supplement 2}
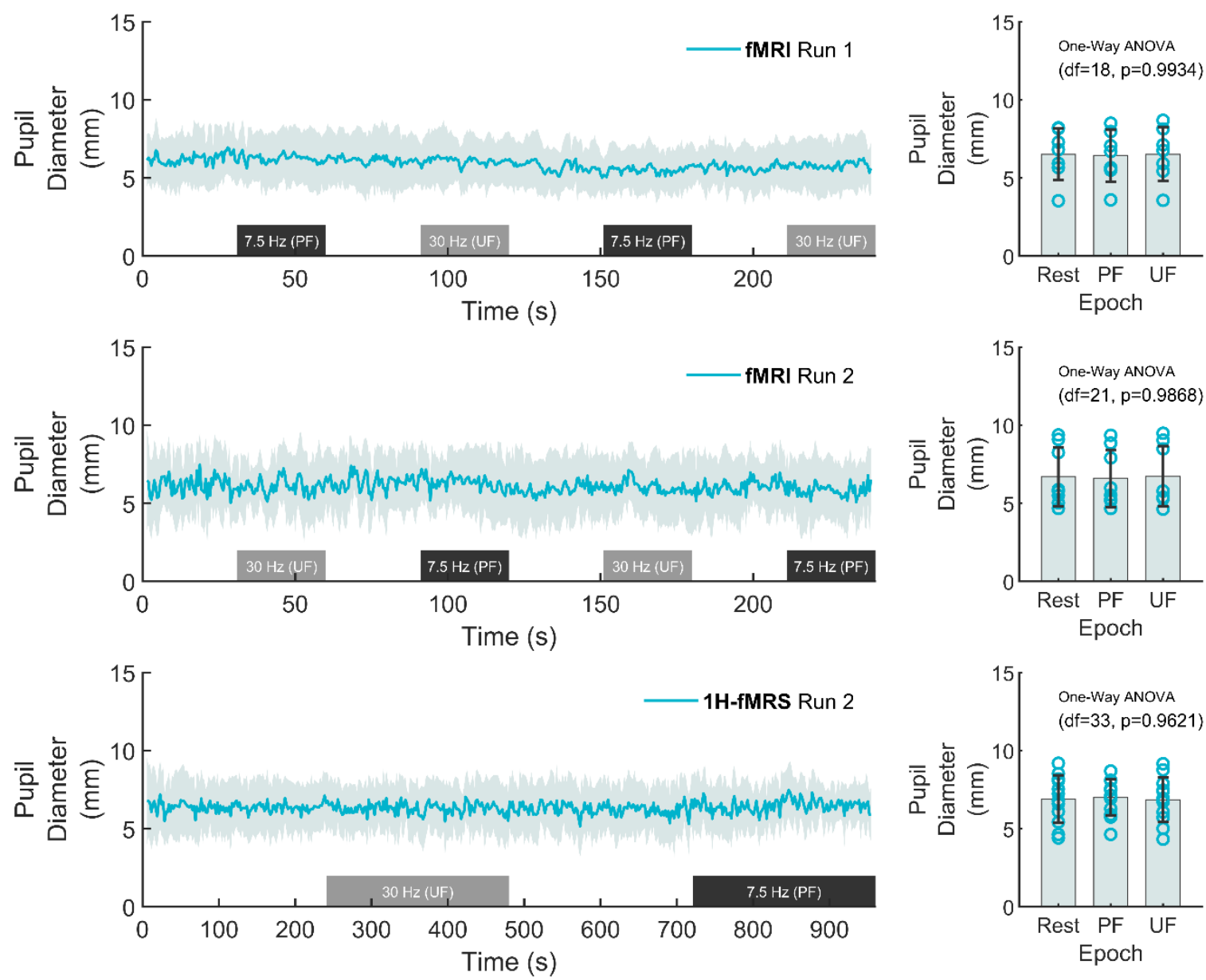

Left: average pupil diameter (across-subjects) during a representative session (1H-fMRS Run 1). Right: mean pupil diameter was not statistically different across conditions (One-Way ANOVA, $\mathrm{p}>0.96$ ). Error bars correspond to SD. Sessions are shown in different rows (Top: fMRI Run 1; Middle: fMRI Run 2; Bottom: 1H-fMRS Run 2). 
Figure 1 - figure supplement 3
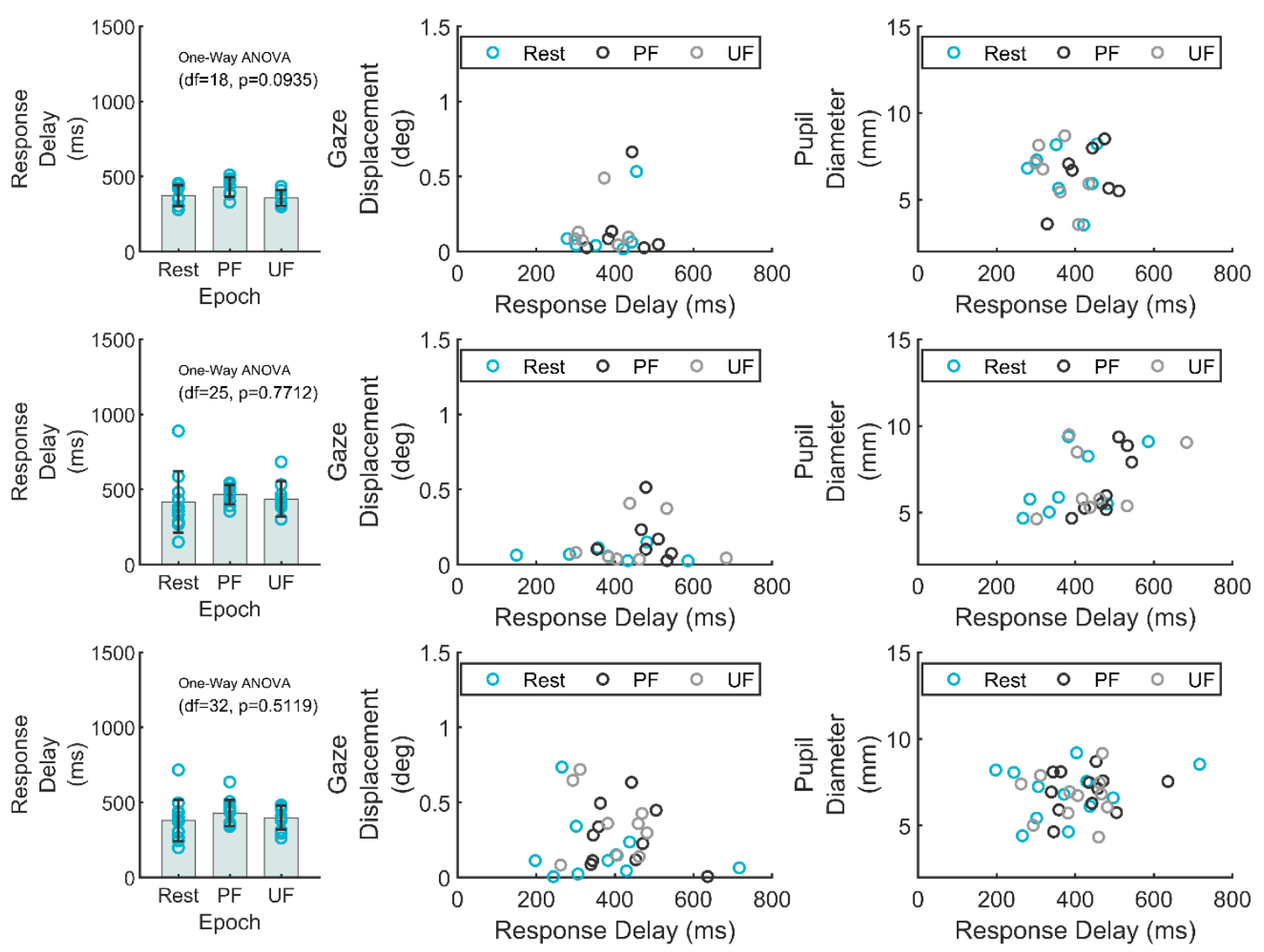

Left: task performance in terms of response delay was not statistically different across conditions (One-Way ANOVA, $\mathrm{p}>0.09$ ). Error bars correspond to SD. Center: there was no correlation between task performance and mean gaze displacement $\left(r^{2}<0.06, p>0.47\right)$. Right: there was no correlation between task performance and mean pupil diameter $\left(r^{2}<0.08, p>0.49\right)$. Sessions are shown in different rows (Top: fMRI Run 1; Middle: fMRI Run 2; Bottom: 1H-fMRS Run 2). 
Figure 2 - figure supplement 1
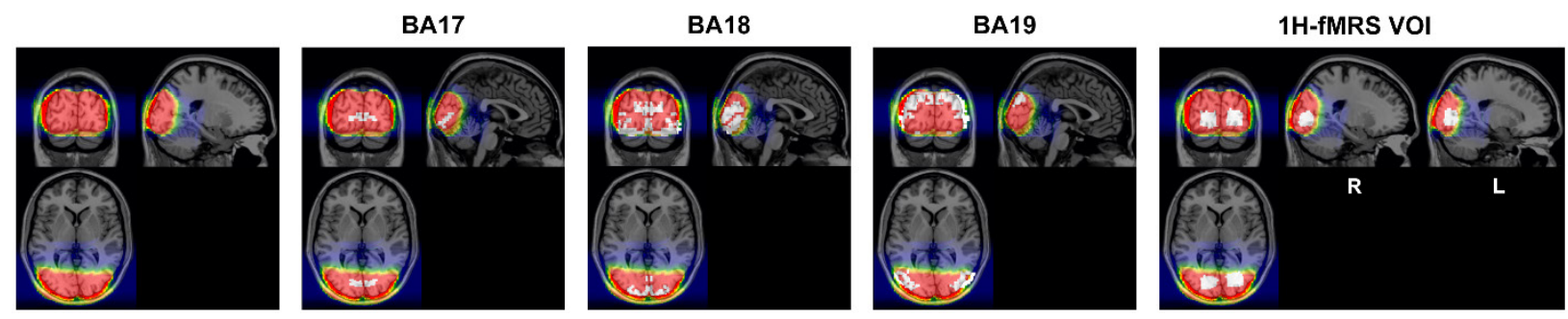

Intensity (\% Peak)

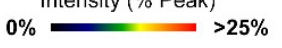

From Left to Right: intensity map of EPI images acquired with the surface coil normalized to MNI template, superimposed to different ROIs (in white): BA17 (i.e., V1), BA18, BA19, and the average spectroscopic voxel (1H-fMRS VOI). 


\section{Figure 4 - figure supplement 1}

$\operatorname{REST}(\mathrm{N}=32)$

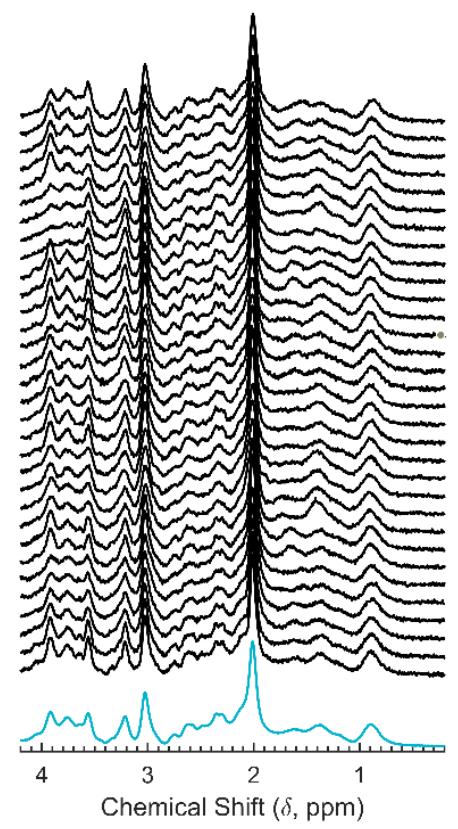

$\mathrm{PF}(\mathrm{N}=16)$

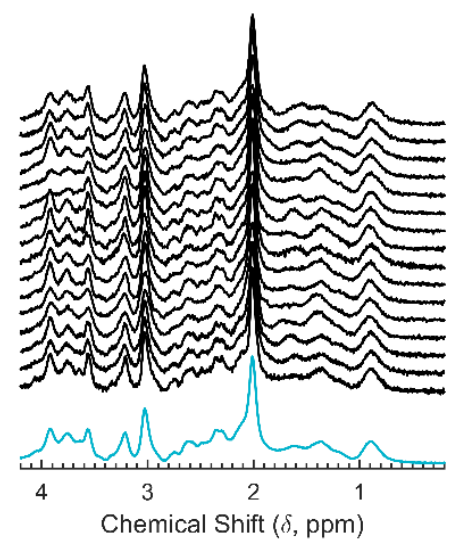

UF $(\mathrm{N}=16)$

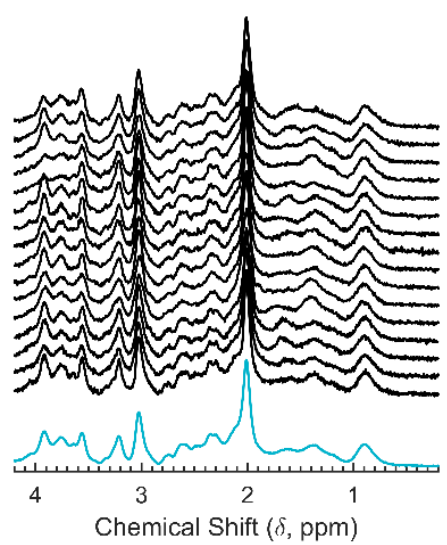

Averaged 1H-fMRS spectra across individual epochs (Left: REST; Center: PF; Right: UF) of the subset of subjects $(\mathrm{N}=16)$ who had a reliable quantification for both Lac and Glu.

$<$ insert Table S1 here followed by a page break $>$ 
bioRxiv preprint doi: https://doi.org/10.1101/2021.09.17.460759; this version posted September 20, 2021. The copyright holder for this preprint (which was not certified by peer review) is the author/funder, who has granted bioRxiv a license to display the preprint in perpetuity. It is made available under aCC-BY 4.0 International license.

\section{Movie S1 (separate file).}

The movie shows the pupil of a representative subject during a stimulation cycle including UF and PF epochs, as well as the relevant physiological and behavioral responses. 